\title{
Parental characteristics and offspring mental health and related outcomes: a systematic review of genetically informative literature
}

\author{
Eshim S. Jami 1,2, Anke R. Hammerschlag ${ }^{1,3,4}$, Meike Bartels (1) ${ }^{1,3}$ and Christel M. Middeldorp (1] ${ }^{1,4,5}$
}

\begin{abstract}
Various parental characteristics, including psychiatric disorders and parenting behaviours, are associated with offspring mental health and related outcomes in observational studies. The application of genetically informative designs is crucial to disentangle the role of genetic and environmental factors (as well as gene-environment correlation) underlying these observations, as parents provide not only the rearing environment but also transmit 50\% of their genes to their offspring. This article first provides an overview of behavioural genetics, matched-pair, and molecular genetics designs that can be applied to investigate parent-offspring associations, whilst modelling or accounting for genetic effects. We then present a systematic literature review of genetically informative studies investigating associations between parental characteristics and offspring mental health and related outcomes, published since 2014. The reviewed studies provide reliable evidence of genetic transmission of depression, criminal behaviour, educational attainment, and substance use. These results highlight that studies that do not use genetically informative designs are likely to misinterpret the mechanisms underlying these parent-offspring associations. After accounting for genetic effects, several parental characteristics, including parental psychiatric traits and parenting behaviours, were associated with offspring internalising problems, externalising problems, educational attainment, substance use, and personality through environmental pathways. Overall, genetically informative designs to study intergenerational transmission prove valuable for the understanding of individual differences in offspring mental health and related outcomes, and mechanisms of transmission within families.
\end{abstract}

\section{Introduction}

Parents are considered a driving force in the development of their children and parental factors are associated with various mental health outcomes in offspring, including emotional and behavioural problems. ${ }^{1}$. However, although observed associations between parental factors and offspring outcomes are often interpreted as direct environmental influences, in truth parents provide both the rearing environment and genes to their children.

\footnotetext{
Correspondence: Eshim S. Jami (e.shahid@ucl.ac.uk)

'Department of Biological Psychology, Vrije Universiteit Amsterdam, Amsterdam, the Netherlands

${ }^{2}$ Department of Clinical, Educational and Health Psychology, Division of Psychology and Language Sciences, University College London, London, UK Full list of author information is available at the end of the article
}

Thus, observed parent-offspring associations may be wholly or partially explained by genetic factors shared between the parent and child; i.e. in a gene-environment correlation $(r G E)$, when exposure to specific environments depends on an individual's genotype. The potential mechanisms (genetic transmission, environmental transmission and gene-environment correlation) underlying associations between parental characteristics and offspring outcomes are described in detail in Fig. 1. Designs that do not account for the role of genetic factors in parent-offspring correlations can lead to biased estimates and erroneous conclusions about the extent to which these associations are causal. Genetically informative designs that explicitly model or control for potential genetic effects are essential for

\section{(c) The Author(s) 2021}

(c) (i) Open Access This article is licensed under a Creative Commons Attribution 4.0 International License, which permits use, sharing, adaptation, distribution and reproduction cc) in any medium or format, as long as you give appropriate credit to the original author(s) and the source, provide a link to the Creative Commons license, and indicate if changes were made. The images or other third party material in this article are included in the article's Creative Commons license, unless indicated otherwise in a credit line to the material. If material is not included in the article's Creative Commons license and your intended use is not permitted by statutory regulation or exceeds the permitted use, you will need to obtain permission directly from the copyright holder. To view a copy of this license, visit http://creativecommons.org/licenses/by/4.0/. 


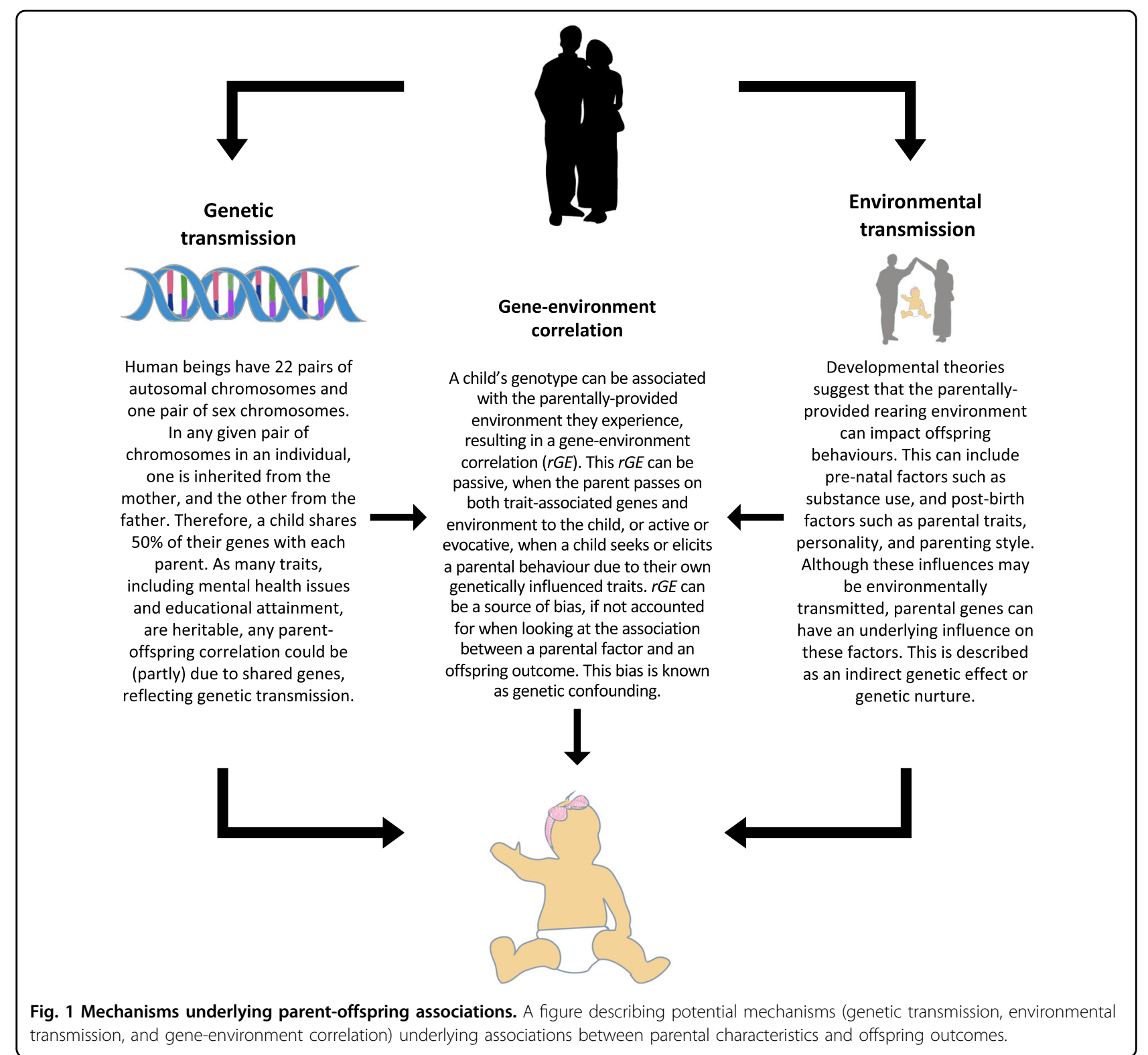

improving our understanding of the true effect of the parentally provided environment on offspring mental health.

In genetic epidemiology, the classical twin design is generally used to decompose the contribution of genetic and environmental effects underlying human traits $^{2}$. Twin-based research shows that most mental health and related traits are moderately heritable (under the influence of additive genetic effects), with additional variance explained by the unique environment (which is specific to each individual), and for some traits also the shared environment (environments that the twins have in common) $)^{3}$. However, classical twin studies say little about mechanisms of transmission within families where, in addition to genetic transmission, parental effects may be transmitted through both the shared environment via parentally provided rearing factors, and to a lesser extent, the unshared environment through specific parent-child interactions. Consequently, genetically informative designs that include both the parent and offspring generations are required to disentangle genetic and environmental effects underlying parent-offspring associations.

The present review aims to synthesise literature investigating the association between parental characteristics and offspring mental health and related outcomes in genetically informative designs. An earlier systematic review published in 2014 focused on the children-of-twins $\operatorname{method}^{4}$. However, several novel methodologies that investigate within-family transmission using innovative techniques have emerged in the past few years. 
Consequently, there is a gap in the literature for a broad systematic overview that incorporates all genetically informative designs that can be applied to study parent-offspring associations. Here, we focus on studies published from 2014 onwards, as these have not been covered by previous reviews. We first provide a brief overview of the types of genetically informative designs that can be employed to investigate parent-child associations. This is followed by a systematic review of studies investigating associations between parental characteristics and offspring mental health and related outcomes, including internalising behaviours (such as anxiety and depression), externalising behaviours (such as attentiondeficit/hyperactivity disorder), educational attainment, substance use and personality.

\section{Genetically informative designs}

Designs that can be used to separate genetic and environmental mechanisms of transmission from parents to offspring broadly fall into the following three categories: behavioural genetics designs, matched-pair designs, and molecular genetics designs. In this section, we summarise the principles underlying these approaches (Fig. 2), describe specific methods in detail and discuss their application as well as advantages and disadvantages (Table 1).

\section{Behavioural genetics designs}

Behavioural genetics designs leverage knowledge of relatedness among individuals within a family to make inferences about the contribution of genetic and environmental factors underlying parent-offspring associations. The adoption ${ }^{5}$ and children-of-twins ${ }^{4,6}$ designs (Fig. 2) are key tools used to distinguish the effects of genetic and environmental transmission. Associations between biological parents and their adopted-away offspring suggest genetic transmission as although these parents and offspring are genetically related, the parents do not raise the child and hence have no environmental influence. On the other hand, associations between adoptive parents and offspring suggest environmental transmission as these parents and offspring are genetically unrelated, and are only connected through the environment. In children-oftwins studies, children of monozygotic twins are as genetically similar to their twin aunt/uncle as they are to their twin parent, whereas children of dizygotic twins share less genetic similarity with their aunt/uncle. Higher monozygotic than dizygotic avuncular correlations (between uncle/aunt and niece/nephew) are likely due to the higher proportion of shared genes, suggesting genetic transmission, whereas higher parent-offspring than monozygotic or dizygotic avuncular correlation indicates environmental transmission through the shared parent-child environment. Another key characteristic of adoption and children-of-twins studies is that they can be used to investigate $r G E$ (Table 1). This is particularly important as even within genetically informative designs, unmeasured $r G E$ can inflate estimates of genetic or environmental effects. For instance, if an observed parent-offspring association is present in both biological and adoptive duos, but the correlation is higher in biological (shared genes plus rearing) than adoptive (rearing only) families, this indicates the contribution of both genetic and environmental effects; i.e. passive $r G E$. If unaccounted for, this $r G E$, reflected in increased similarity between biological parents and lived-with offspring, could potentially lead to an inflated estimation of genetic transmission in adoption studies.

Due to modern developments in assisted reproductive technology and the availability of large-scale populationbased registers, novel pseudo-adoption designs have emerged that apply the same principles (see adoption and related designs in Fig. 1) to investigate genetic and environmental effects in non-adoption families. Within assisted conception ${ }^{7}$ studies, genetically related or genetically unrelated parents are analogous to the biological and adoptive parents in an adoption design, whereas in triparental family ${ }^{8}$ and multiple parenting relationships ${ }^{9}$ designs, the rearing effect of step-parents and genetic effect of not-lived-with biological parents are examined (Table 1).

\section{Matched-pair designs}

Matched-pair designs strengthen the causal inference of an observed parent-offspring association by adjusting for all unmeasured genetic and environmental familial effects. In sibling comparison ${ }^{10}$ studies (Fig. 2), a sibling with no exposure to the parental candidate environment is included in the analysis as a control, as siblings are naturally matched for shared genes and the family environment. Environmental transmission is indicated if the parent-offspring association is observed only in the exposed offspring. Similarly, the case-control ${ }^{11}$ design includes matched parent-child control pairs who share the same proportion of genetic and environmental factors as the case parent-child pairs, but do not share the candidate exposure. As the matching is done by the researchers here, it is crucial that the process is thorough so that it can be reasonably argued that unmeasured confounders are unlikely to bias the results. Matched-pairs designs cannot be used to investigate $r G E$, as they do not directly measure genetic effects. However, sibling comparison studies generally rule out passive $r G E$, as the random distribution of parental alleles across offspring ensures that siblings are equally likely to receive genes associated with the exposure in the parent, and the outcome in the offspring. Evocative $r G E$ can also be ruled out if exposure to the parental characteristic definitively precedes the offspring outcome. 


\section{A. Behavioural genetics designs}

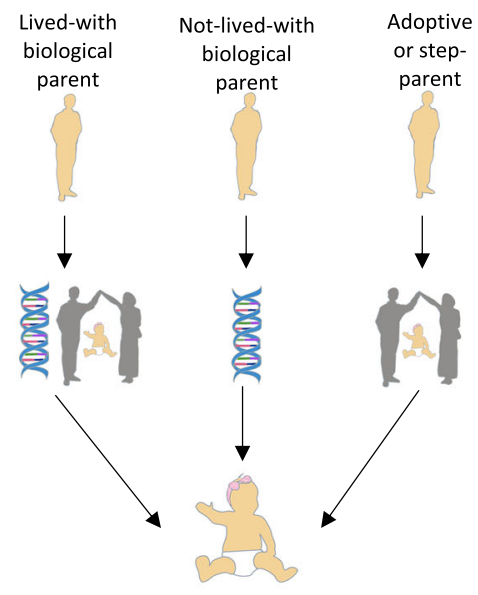

Adoption and related studies

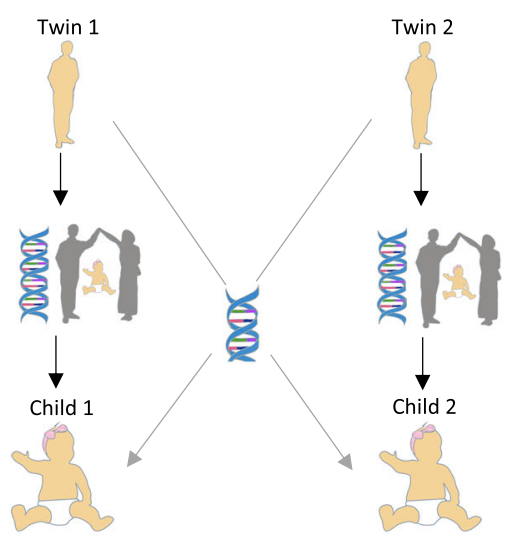

Children-of-twin studies

\section{B. Matched-pair designs}

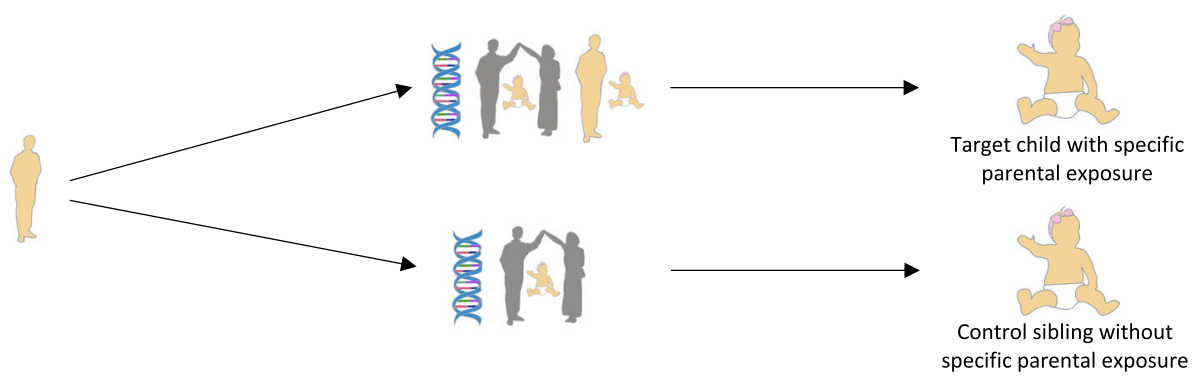

Sibling comparison studies

\section{Molecular genetics designs}

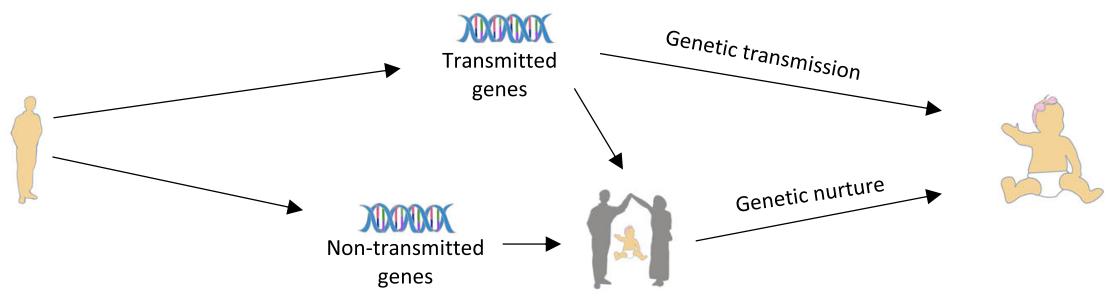

Fig. 2 Schematic diagrams demonstrating the principles underlying commonly used genetically informative designs which separate genetic and environmental mechanisms of transmission in parent-offspring associations. A In adoption and related designs, knowledge of the type of relationship shared between parent and offspring is leveraged to gain insight into genetic and environmental factors underlying parentto-offspring associations. Lived-with biological parents can influence offspring through both genetic and environmental transmission, as they provide both genes and the rearing environment. Not-lived-with biological parents who have no contact with the offspring provide only genes, indicating genetic transmission, whereas adoptive or step-parents provide only the rearing environment, indicating environmental transmission. In children-oftwins studies, children of identical (monozygotic) twins are as genetically similar to their aunt/uncle as they are to their parents (50\% shared genes), whereas children of fraternal (dizygotic) twins share $25 \%$ of genes with their aunt/uncle. Higher monozygotic than dizygotic avuncular correlations (between uncle/aunt and niece/nephew, i.e. between Twin 1 and Child 2 or Twin 2 and Child 1) are likely due to a higher proportion of shared genes, suggesting genetic transmission, whereas higher parent-offspring than avuncular correlation suggests environmental transmission of a parental factor, above and beyond the effect of shared genetic or environmental effects. B In sibling comparison studies, the association between a specific parental factor and offspring outcome is studied in exposed versus unexposed offspring, as siblings are naturally matched for parentally provided genes and a rearing environment. Environmental transmission is indicated if the parent-offspring association is observed only in the exposed offspring. C In molecular genetics studies, the effect of shared parent-offspring (i.e. transmitted) genes on offspring outcome indicates the presence of genetic transmission. However, both transmitted genes and non-transmitted parental genes can also have an indirect (i.e. environmentally mediated) effect on offspring through parental traits that are genetically influenced; this is otherwise known as genetic nurture. 


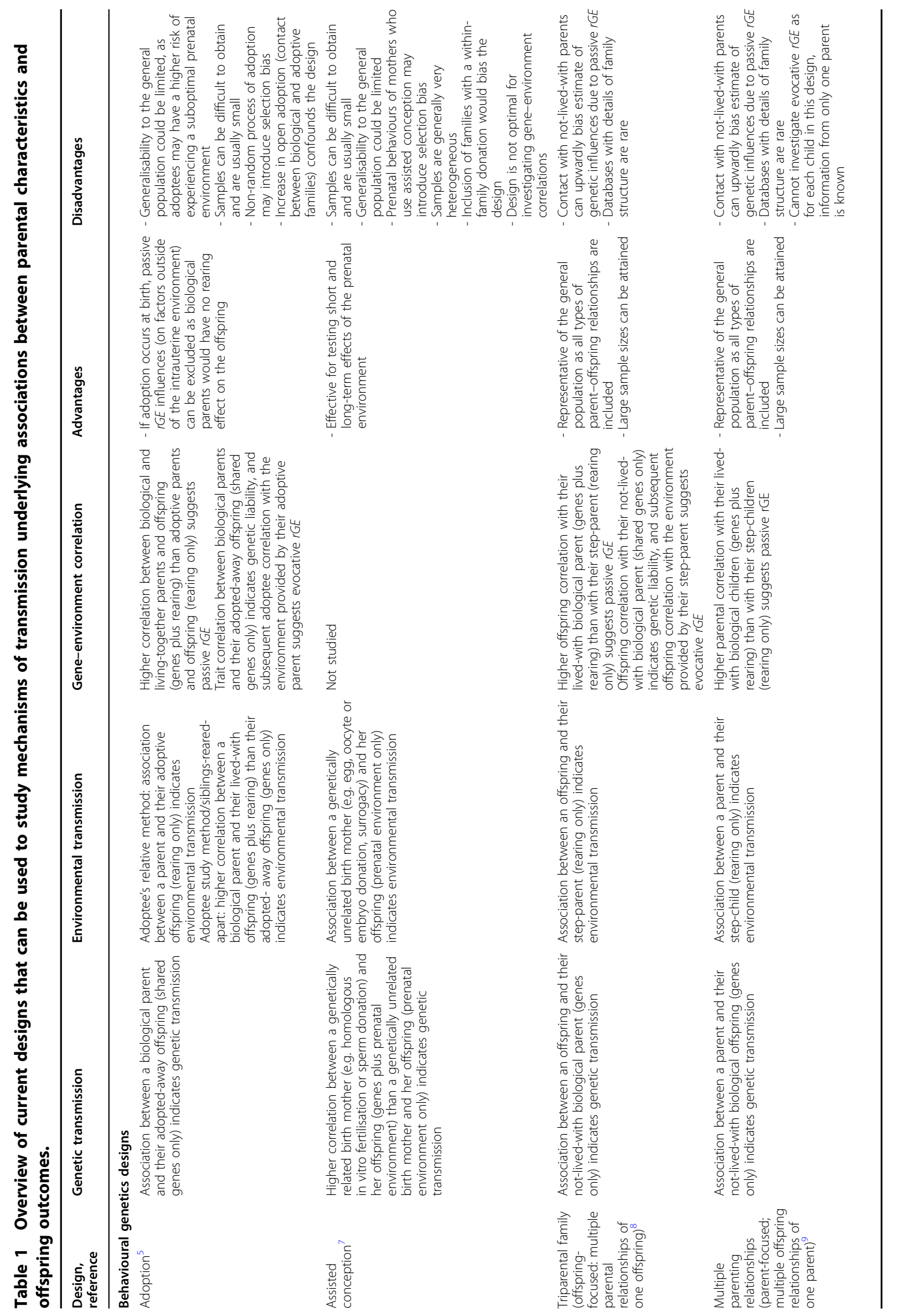




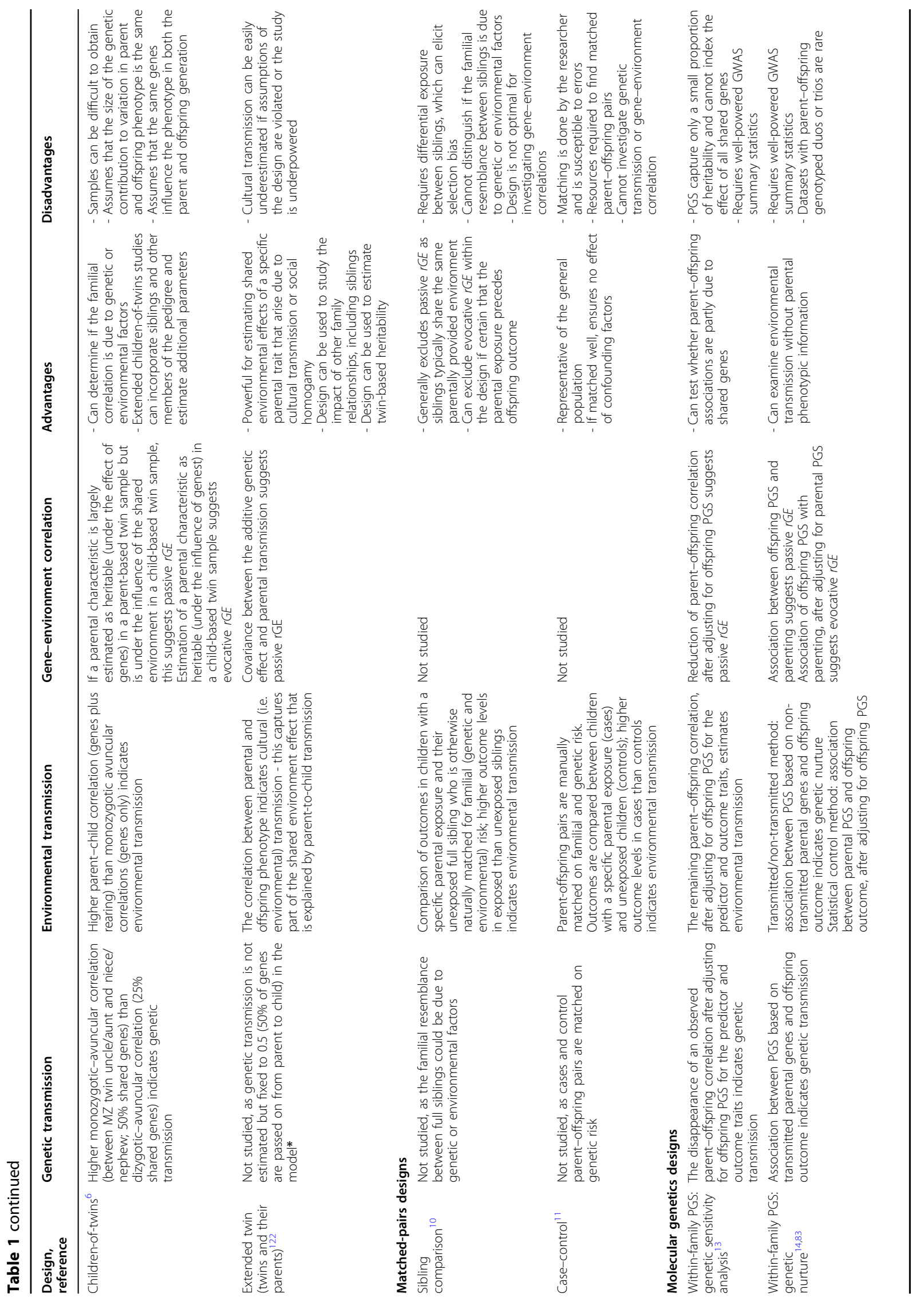




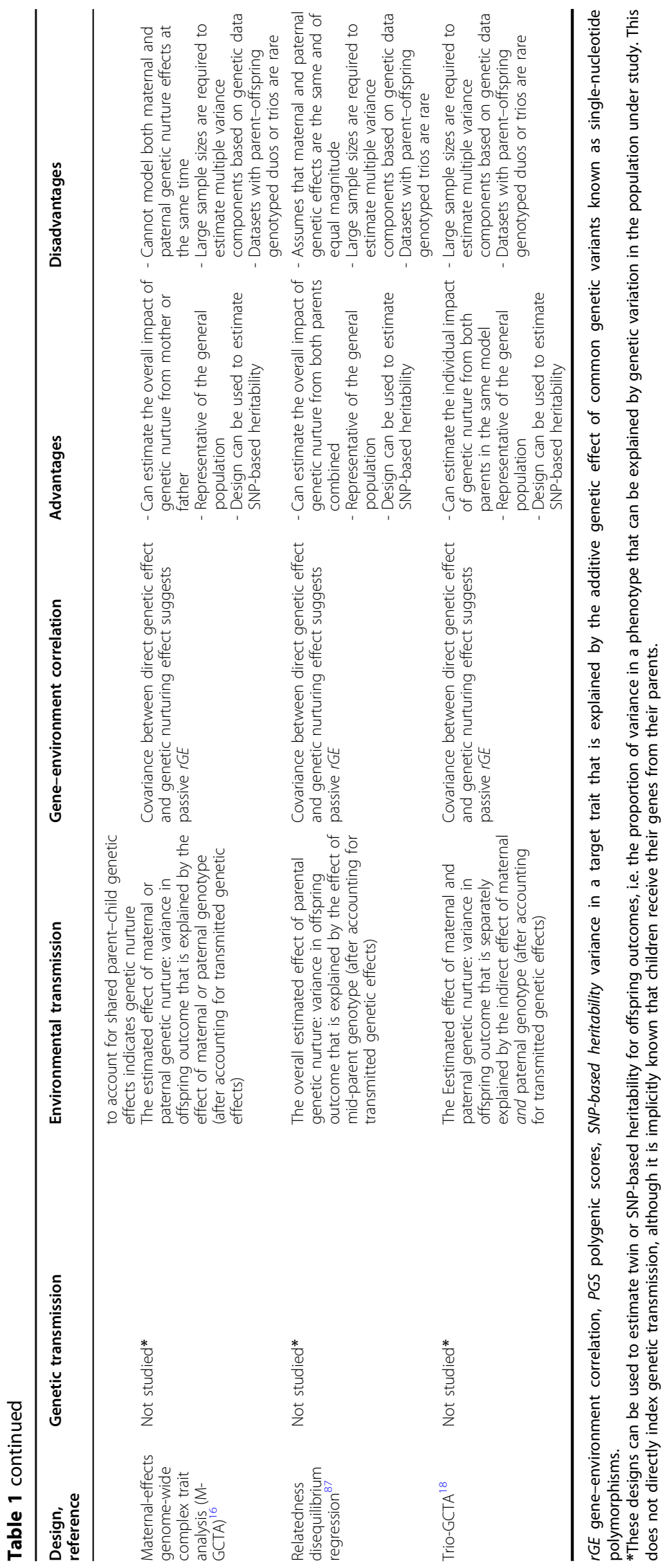




\section{Molecular genetics designs}

Recent advances in molecular genetics provide novel ways of investigating genetic and environment effects underlying parent-offspring associations by using genomic data. In molecular genetics studies, the effect of genetic variants transmitted from parent-to-offspring on offspring behaviour indicates the presence of genetic transmission. As described in Figs. 1 and 2, parental genes can also have an indirect effect on offspring, through parental traits that are environmentally mediated but genetically influenced; a process otherwise known as genetic nurture. One way to separate genetic transmission and genetic nurture effects underlying specific parent-offspring associations is the use of polygenic scores. Polygenic scores (PGS) represent an aggregate genetic liability for a trait, determined by the presence and effect sizes of alleles associated with the trait ${ }^{12}$. In withinfamily PGS genetic sensitivity analysis, offspring PGS for exposure and outcome traits are included as covariates in the regression analyses to explore whether the association between a parental exposure variable and offspring outcome is attenuated by the offspring's PGS. If that is the case, genetic transmission explains part of the parent-offspring association ${ }^{13}$. Although adjusting for PGS does not entirely eliminate genetic transmission as current PGS capture only a small proportion of trait heritability, such sensitivity analyses can show whether shared genes partially account for an observed parent-offspring association. In within-family PGS genetic nurture analyses, PGS can additionally be used to estimate the environmental influence of parental alleles not passed on to the offspring ${ }^{14,15}$. If PGS based on nontransmitted parental alleles are associated with an offspring trait (transmitted/non-transmitted method in Table 1), the effect of these parental genes on offspring behaviour likely occurs via an environment pathway, i.e. genetic nurture. Similarly, if parental PGS are associated with an offspring trait, after adjusting for the child's own PGS (statistical control method in Table 1), this also suggests a nurturing effect of parental genes beyond that which is due to transmitted genes (see statistical control method in Table 1). The overall contribution of genetic nurture to offspring traits can be estimated using maternal-effects genome complex trait analysis (M-GCTA) ${ }^{16}$, relatedness disequilibrium regression $(\mathrm{RDR})^{17}$ or trio-GCTA ${ }^{18}$ (Table 1). Each of these methods uses genotyped data from unrelated parent-offspring pairs to estimate the variance in offspring behaviour that is explained by their own genotype (SNP-based heritability; heritability accounted for by differences in measured genetic variants known as single-nucleotide polymorphisms) and genetic nurture (parental additive genetic effects acting via genetically influenced parental behaviours).

It is important to note that as current genetic nurture designs only index parental effects that are captured by their common genetic variation, these designs capture only a part of the overall parent-to-child environmental transmission. Parental traits that are not under the influence of common genetic variation may also influence offspring outcomes. To test whether specific parental behaviours are responsible for observed genetic nurturing effects, the parental phenotype can be included as a covariate in within-family genetic nurture analyses, $\mathrm{M}$ GCTA, RDR or trio-GCTA. If a genetic nurturing effect on offspring behaviour is attenuated with the inclusion of the parental phenotype to the model, the parental phenotype is shown to be involved in the manifestation of the genetic nurturing effect. As with behavioural genetics designs, molecular genetics designs can be used to investigate $r G E$, by estimating covariance between additive genetic effects and indirect genetic nurturing effects (Table 1).

\section{Methods}

We searched for articles investigating associations between parental characteristics and offspring mental health and related outcomes. We defined related traits as those that have an established link to mental health in the literature. The Web of Science database was used to conduct a systematic search of studies published from 2014 to June 2020. The search terms consisted of study design variables ("children-of-twins" or "offspring of twins" or "adoption" or "assisted conception" or "sibling comparison" or "genetic nurture" or "non-transmitted" or "polygenic score"), parent variables ("parent" or "mother" or "father" or "maternal" or "paternal"), offspring variables ("offspring" or "child") and topic variables ("gene" or "environment"). The search did not include predictor or outcome-specific search terms, so as not to limit the review to a particular set of traits. We restricted the search to scientific articles published in English. Through the results of the initial search, we identified additional designs that were relevant (Table 1), and ran separate follow-up searches for these study design variables ("extended twin" or "triparental" or "multiple parenting relationships design" or "matched pair" or "genome-wide complex trait analyses" or "relatedness disequilibrium regression"). Aside from the database searches, we scanned the references of papers for relevant studies and checked bioRxiv and medRxiv for relevant preprints.

After removing duplicates, the overall search yielded 2097 hits. Studies were included in the systematic review when the following criteria were met: the association between a parental characteristic and offspring behaviour was examined, a genetically informative design was used, and the phenotype in the offspring was a mental health or related trait. As current literature shows that most complex traits have a polygenic architecture, candidate gene studies were excluded from this review. 


\section{Results}

After screening and assessment of search results (Fig. 3), we identified 89 articles for inclusion in this review. We present our synthesis of the literature by grouping the studies according to the offspring outcome in the following sections: internalising behaviours, externalising behaviours, educational attainment, substance use and personality. The number of studies and key findings for each outcome are summarised in Table 2. Details of all studies and their results are reported in Tables 3-7. Effect sizes showing the relative contribution of genetic and environmental factors in parent-offspring associations are included in the tables when studies provided standardised, well-interpretable statistics, i.e., odds ratios, percentage of variance explained or standardised betas.

\section{Offspring internalising behaviours}

\section{Intergenerational transmission of internalising behaviours}

Studies investigating the association between parent and offspring internalising behaviours (Table 3), including depression and anxiety, showed substantial evidence of genetic transmission of depressive symptoms ${ }^{19-22}$, and major depressive disorder (MDD) diagnosis ${ }^{23}$. This is in line with twin literature which shows that depression is a heritable phenotype ${ }^{3}$. After accounting for genetic effects, parental depression was associated with offspring internalising behaviours through environmental pathways, and these associations were observed throughout childhood $^{20,21,24,25}$, adolescence ${ }^{19,26}$, and adulthood ${ }^{23}$. Similarly, associations between parental anxiety and offspring internalising behaviours also showed evidence of environmental transmission across development, from
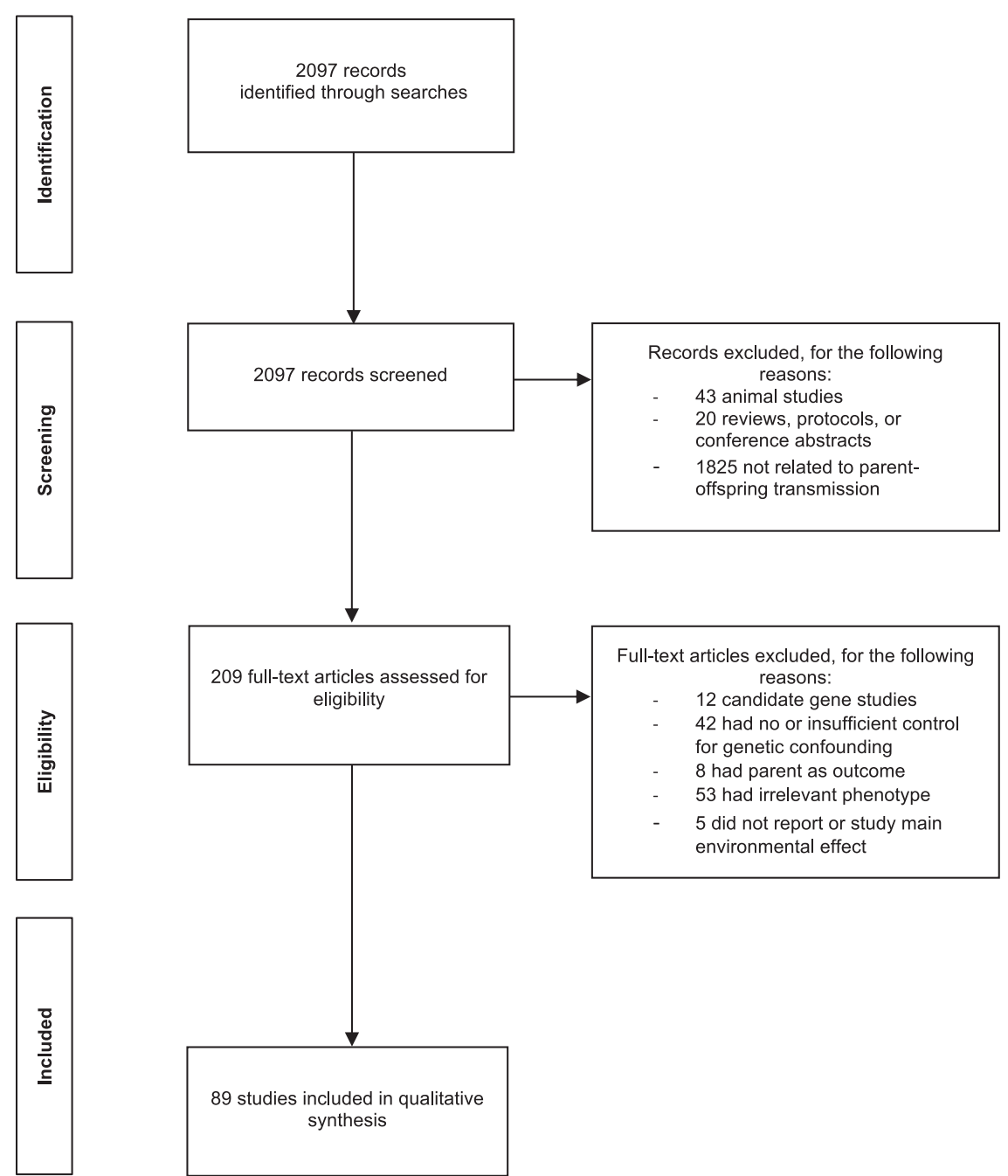

Fig. 3 Flow chart of study selection. A description of the screening and assessment procedure, reporting the number of records excluded and reasons for exclusion at each stage. 


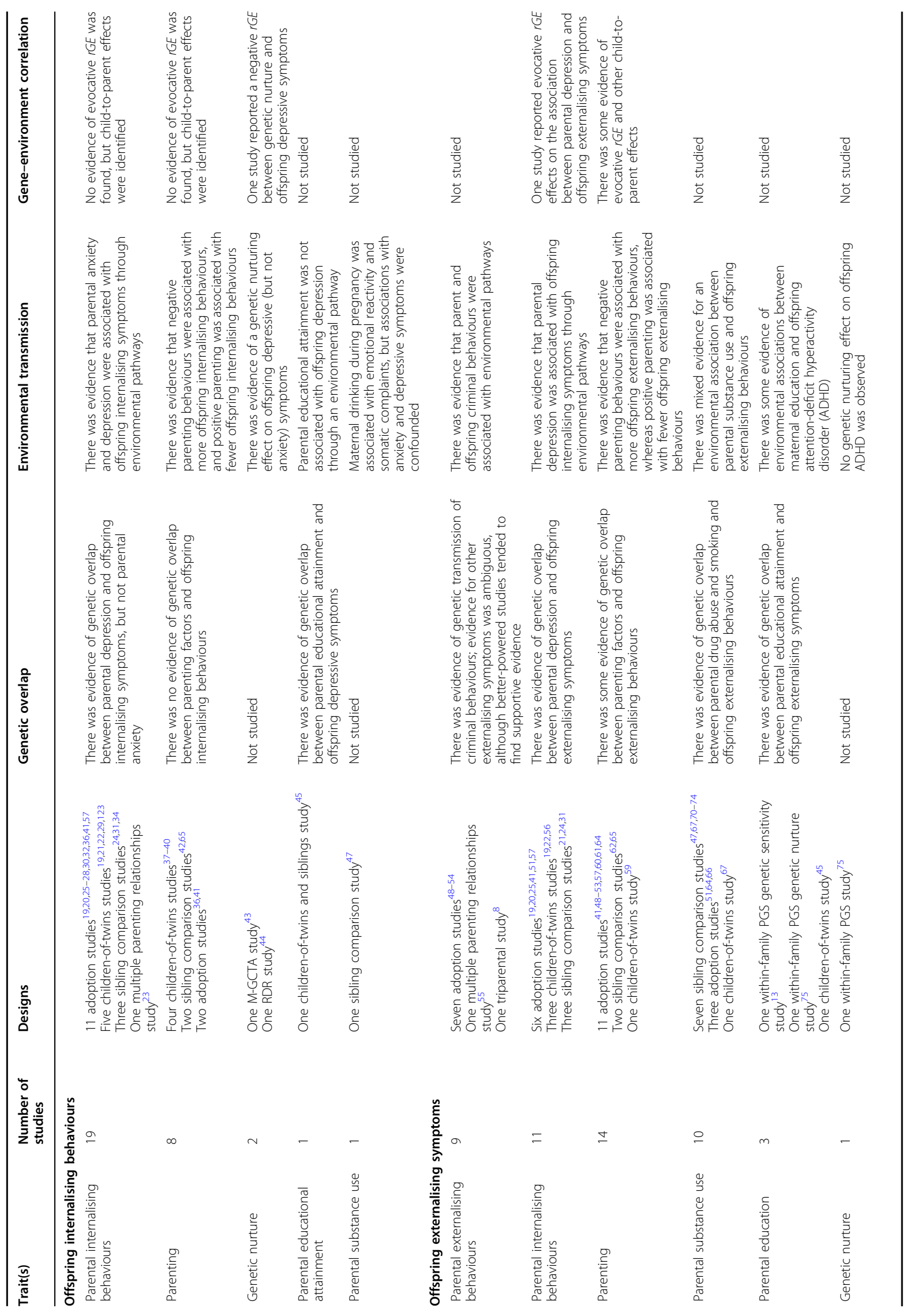




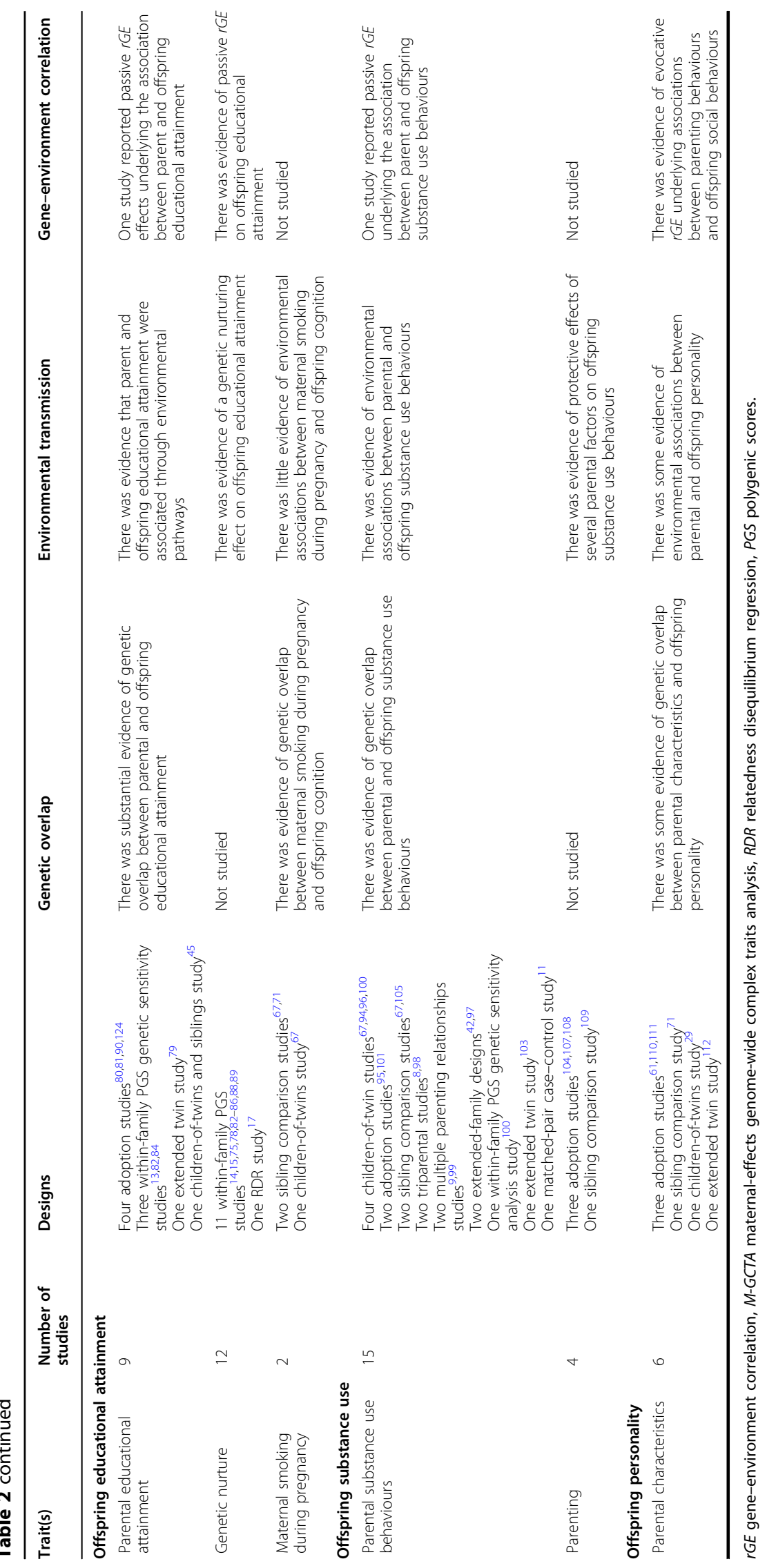




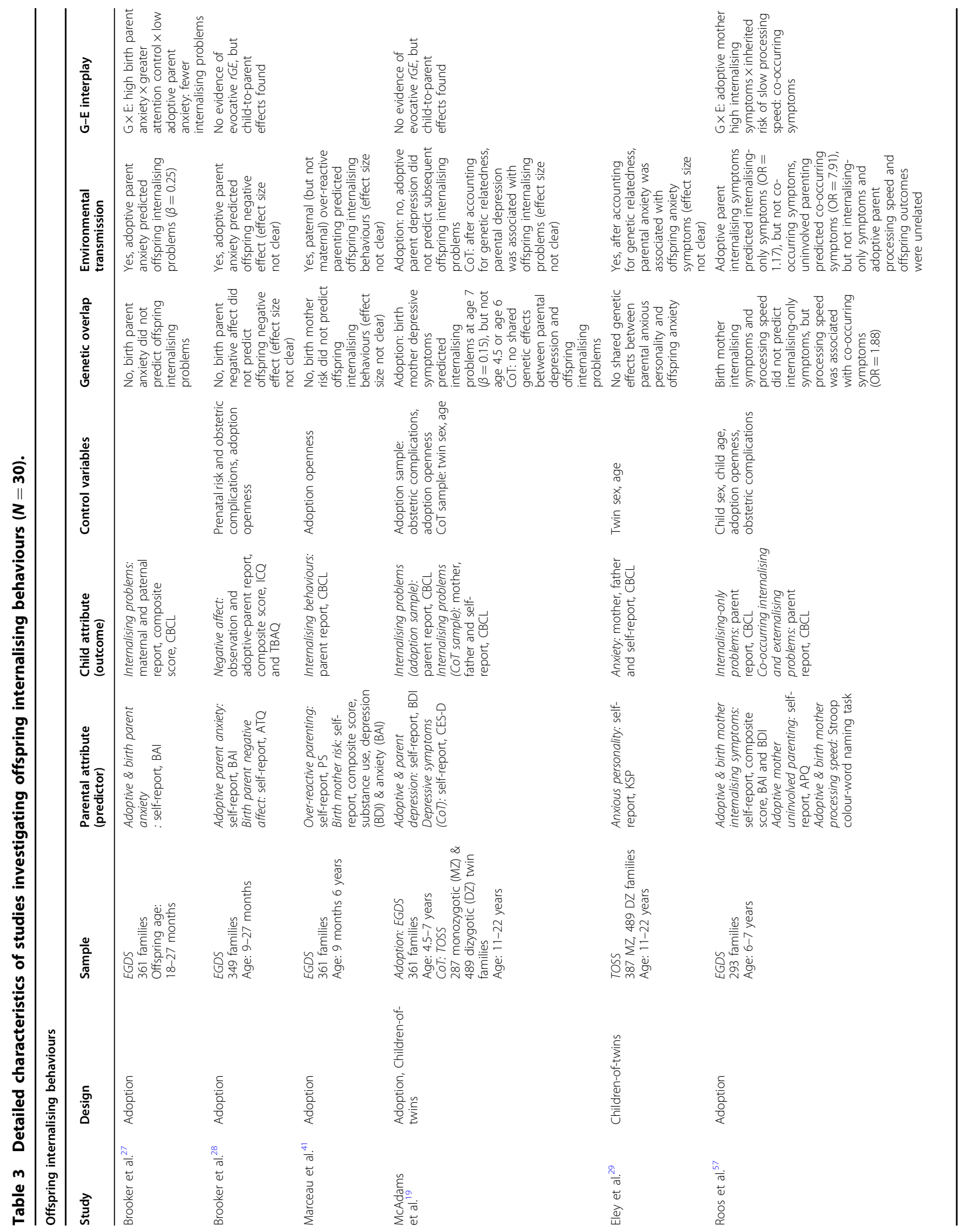




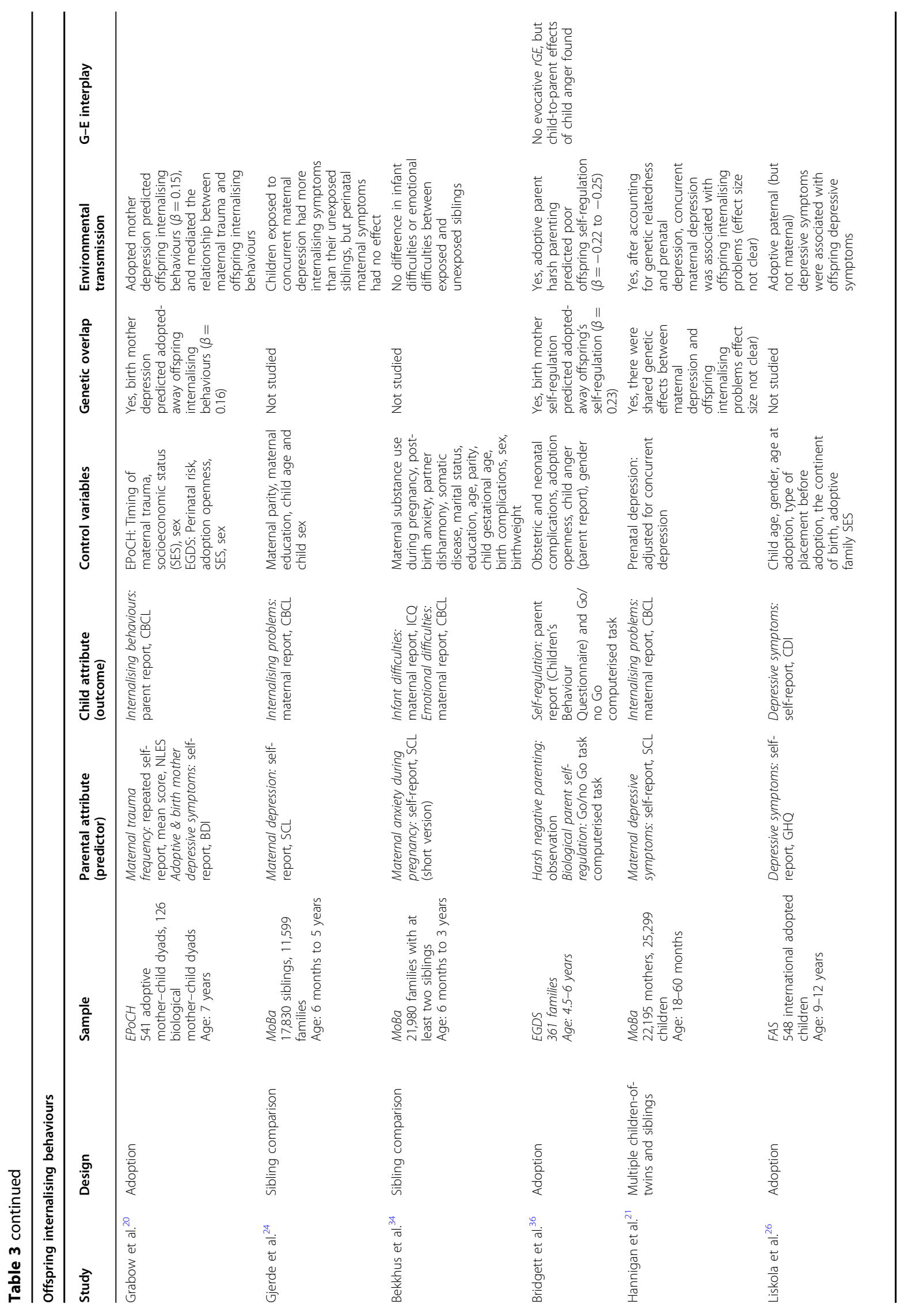




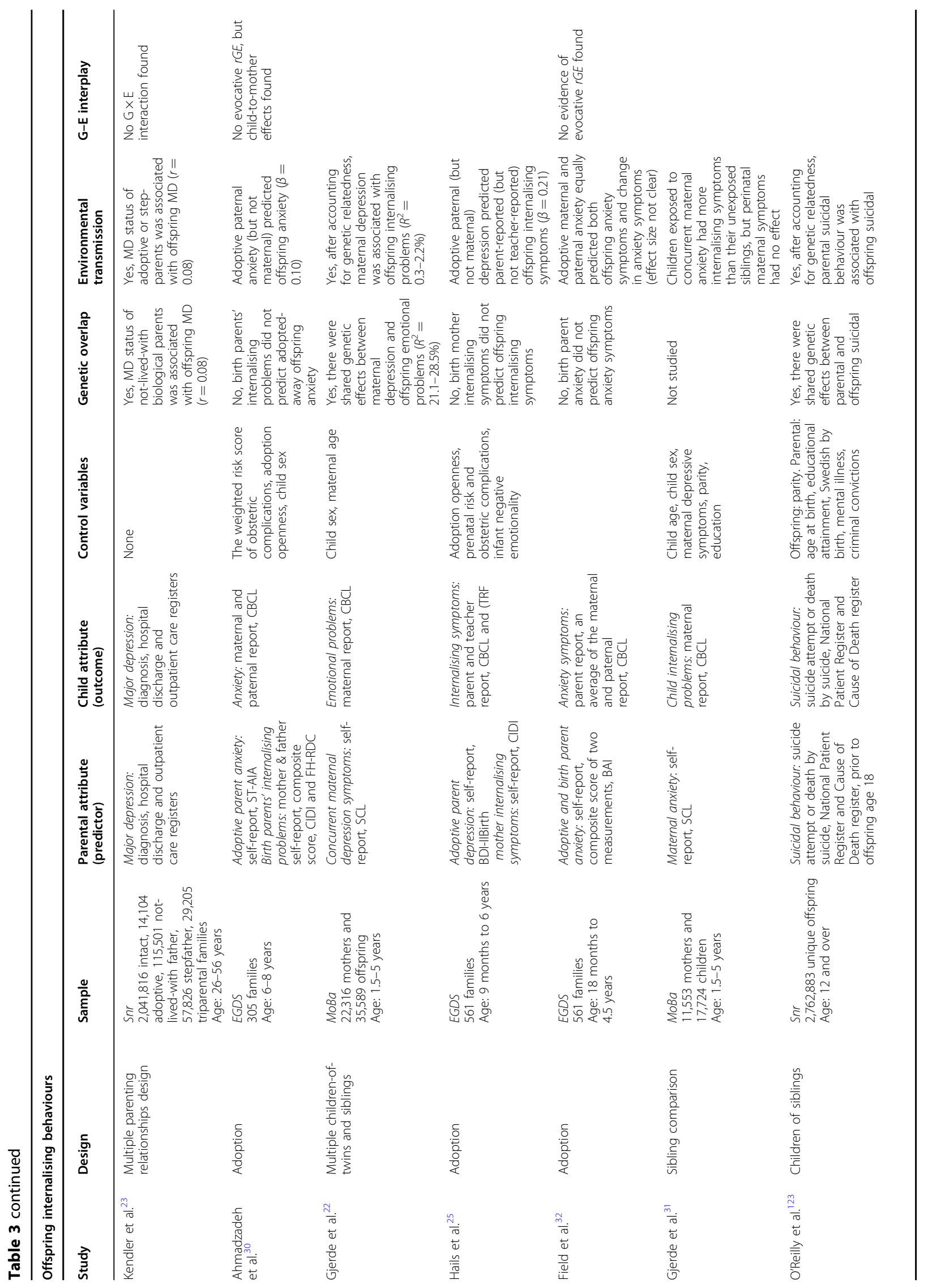




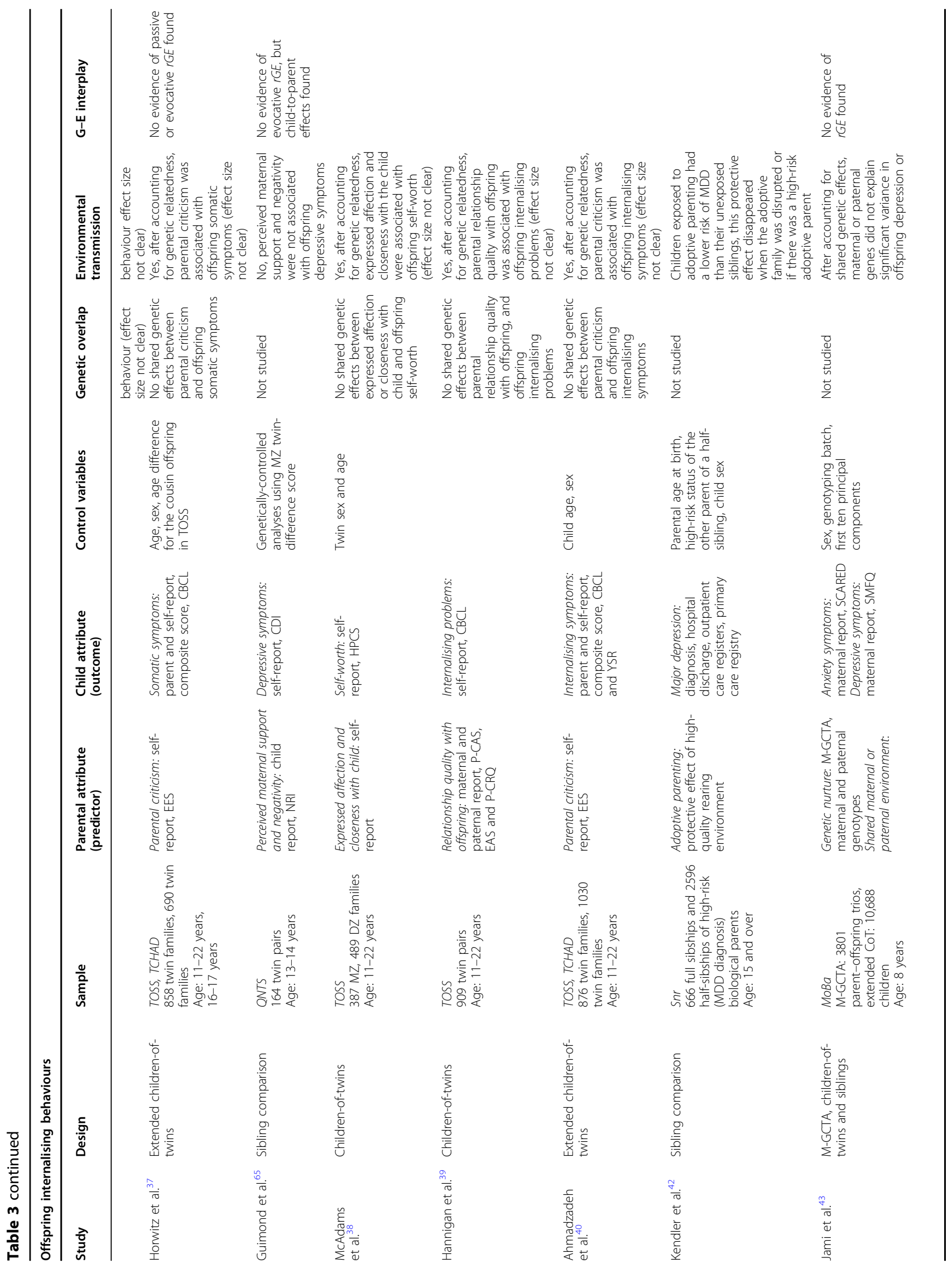




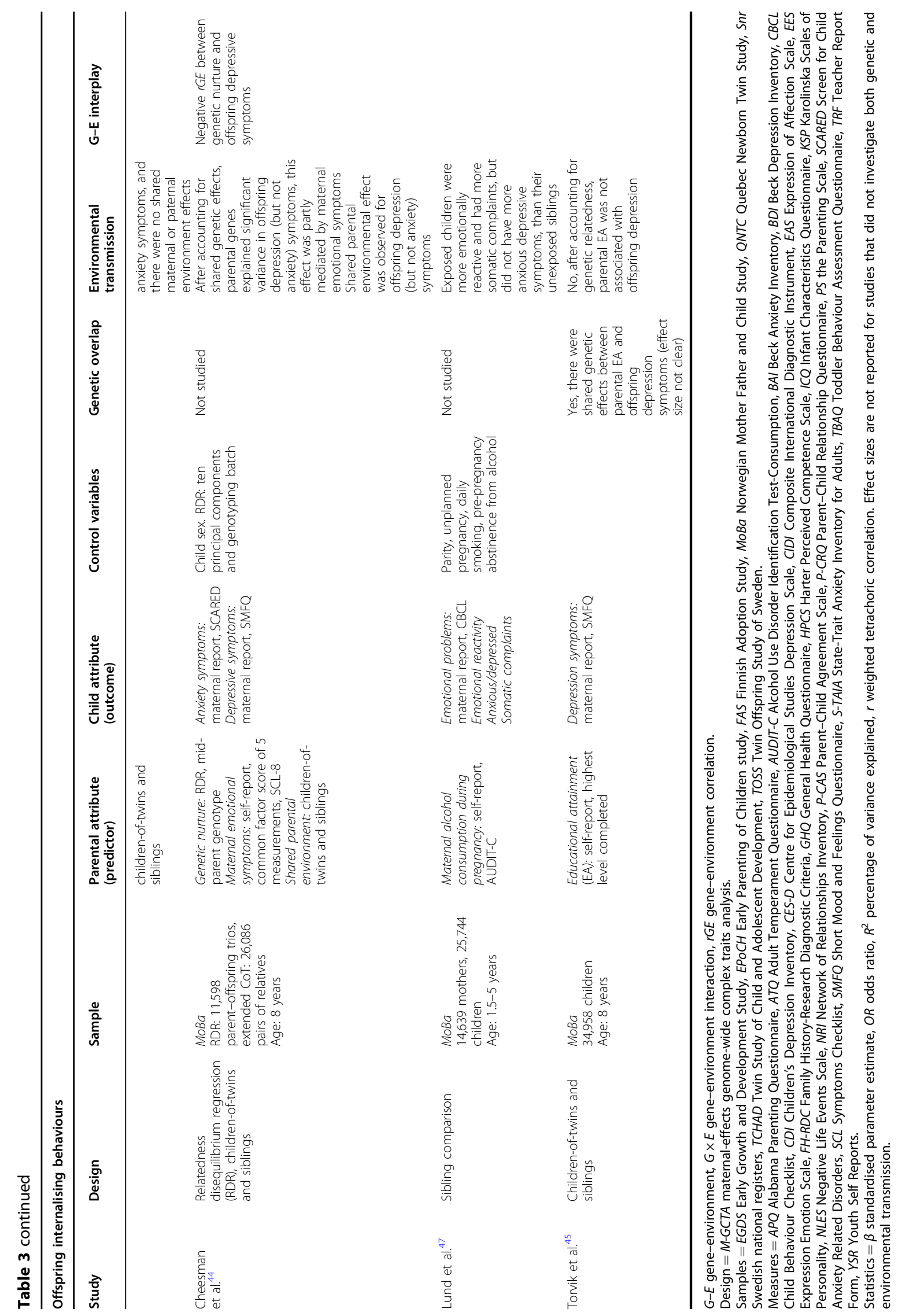


toddlerhood to early adulthood ${ }^{27-32}$. However, unlike depression, this association was not partly explained by shared genes, as there was no evidence of genetic overlap between parental anxiety and offspring internalising behaviours $^{27,29,30,32}$. The lack of evidence for genetic transmission of anxiety is at odds with findings from twin literature which estimate that $40 \%$ of individual differences in anxiety are explained by genetic factors ${ }^{3}$. However, there are some possible explanations of why genetic transmission is not evident within the adoption and children-of-twins studies reviewed here. Measures of inherited risk in the adoption studies could lack validity, and may not adequately capture the genetic risk of anxiety from birth parents. Alternatively, as longitudinal studies show that genetic factors involved in anxiety change across the lifespan ${ }^{33}$, different genes could be relevant for the occurrence of anxiety in early life and adulthood. Therefore, parental anxiety and offspring internalising symptoms may share fewer common genetic factors that are not easily captured using adoption or children-oftwins designs. Even if different genes are involved in childhood internalising symptoms and adult anxiety, the observed environmental association indicates that exposure to an anxious parent is a risk factor for offspring internalising symptoms.

Overall, environmental associations between parental factors and offspring internalising behaviours were generally driven by exposure to concurrent parental anxiety or depression, whereas prenatal and post-natal symptoms did not have a long-lasting effect ${ }^{21,24,31,34}$. This finding stands in contrast to the substantial body of literature that interprets associations between perinatal maternal distress and offspring mental health outcomes in causal terms ${ }^{35}$. Based on the current findings, such parent-offspring associations detected in previous observational studies are likely to be attributable to unmeasured $r G E$, or concurrent parental depression. In investigating the presence of gene-environment correlation, several adoption studies found no evidence of evocative $r G E$, although some child-to-parent effects were identified $^{19,28,30,32,36}$. These studies highlight the dynamic nature of parent and offspring relationships, where associations can be bidirectional, with both parent and offspring behaviour influencing the other.

\section{Parenting behaviours}

Children-of-twin studies examining genetic overlap between parenting and offspring mental health found that genes involved in parenting behaviours (such as parental criticism, parental affection and parent-child relationship quality) did not overlap with genes involved in offspring internalising behaviours ${ }^{37-40}$ (Table 3). After accounting for genetic relatedness, several parenting behaviours were associated with offspring internalising behaviours.
Negative parenting behaviours, including over-reactive parenting $^{41}$, harsh parenting ${ }^{36}$ and parental criticism ${ }^{37,40}$ were associated with more offspring internalising behaviours, whereas parental expressed affection and a good parent-child relationship quality were associated with positive offspring self-worth ${ }^{38}$, and fewer internalising problems $^{39}$, respectively. Of note, an innovative sibling comparison based on Swedish registry data identified a protective effect of adoptive parenting in children of highrisk biological parents with MDD diagnosis ${ }^{42}$. In interpreting associations between parenting behaviours and offspring outcomes, it is important to again note that these parent-offspring associations can be bidirectional, with each affecting the other over time. Furthermore, parenting behaviours can be evoked by the offspring's genetically influenced internalising behaviours. However, three adoption studies found no evocative $r G E$ effects of offspring internalising symptoms ${ }^{32,36,37}$, although one study reported child-to-parent effects wherein child anger predicted prospective harsh negative parenting ${ }^{36}$.

\section{Genetic nurture}

Genetic nurture is a relatively new topic within psychiatric genetics, and as such, we identified only two studies that investigated environmentally mediated effects of parental genes on offspring internalising behaviours (Table 3). Both studies were based on the Norwegian Mother, Father and Child (MoBa) sample and estimated variance in offspring depression and anxiety symptoms that was explained by indirect parental genetic effects, over and above the transmission of genes from parent to child. The earlier study, with a smaller sample size, found no evidence for genetic nurture ${ }^{43}$, whereas the subsequent study with three times the sample size identified a genetic nurturing effect on offspring depressive symptoms that was mediated by maternal emotional symptoms ${ }^{44}$. This finding is in line with the studies reviewed above which showed environmental associations between maternal depression and offspring internalising behaviours ${ }^{20,21,24}$ and shows that seemingly environmental associations between parental factors and offspring outcomes may nonetheless be driven by genetically influenced parental traits.

\section{Parental educational attainment}

A large children-of-twins and siblings study investigating associations between parental educational attainment and offspring depressive symptoms found evidence of genetic, but not environmental transmission ${ }^{45}$ (Table 3). Genetic overlap between education attainment and depression has been reported previously ${ }^{46}$, and this study highlights that without the use of genetically informative designs to account for genetic transmission, phenotypic associations between parental educational attainment and 
offspring internalising symptoms could be misinterpreted as causal.

\section{Parental substance use}

A large sibling comparison study investigated associations between maternal alcohol use during pregnancy and offspring emotional problems ${ }^{47}$ (Table 3). Although exposed children were more emotionally reactive and had more somatic complaints than their unexposed siblings, associations between maternal drinking and offspring anxiety and depressive symptoms seemed to be explained by factors shared by siblings born of the same mother. Previous literature investigating the impact of drinking during pregnancy on offspring internalising behaviours shows mixed findings ${ }^{47}$, making it difficult to make firm conclusions on whether there is an environmental association.

\section{Offspring externalising behaviours Intergenerational transmission of externalising behaviours}

Several adoption studies investigating the intergenerational transmission of externalising behaviours (Table 4) were based on the Early Growth and Development Study (EGDS) sample. Detection of effects in these studies seemed to correlate with sample size, indicating that power considerations are important in interpreting these results. In studies with fewer participants (up to 361 families), birth parent externalising behaviour, antisocial behaviour and self-regulation were uncorrelated with offspring externalising behaviours ${ }^{48-50}$, suggesting no shared genetic effects. However, studies with more participants (561 families) showed correlations between birth parent and offspring externalising behaviours ${ }^{51}$, and between birth parent antisocial behaviour and offspring callous-unemotional behaviours ${ }^{52}$, although oppositional and attentional-deficit behaviours were uncorrelated with birth parent antisociality ${ }^{52}$. Findings from previous literature show substantial heritability of externalising behaviours ${ }^{3}$ and highlight the important role of genetic transmission in explaining parent-offspring simarlity ${ }^{4}$. It is likely that the detection of genetic transmission in adoption studies requires more power, especially if the specific parent and offspring phenotypes under investigation are related, but not identical traits.

The role of environmental transmission in externalising behaviours has also been previously implicated ${ }^{4}$. Here, we identified one adoption study which found no robust evidence for an association between parent antisociality and offspring disruptive behaviours ${ }^{53}$. In addition, three large Swedish population-based studies of criminal behaviour found robust evidence of both genetic and environmental transmission of criminal behaviour ${ }^{8,54,55}$ and showed that risk of criminal behaviour was strongest in families where the same parent provided both the genes and the rearing environment ${ }^{8,55}$.

\section{Parental anxiety or depression}

Evidence from adoption and children-of-twins studies showed genetic overlap between parental depression and offspring externalising behaviours, including ADHD ${ }^{19-22,56}$ (Table 4), whereas associations between overall parental internalising symptoms and offspring externalising symptoms showed mixed results in four adoption stu$\operatorname{dies}^{25,41,51,57}$. Genetic overlap between depression and externalising phenotypes has been reported previously ${ }^{58}$, and the generalist-gene hypothesis suggests that the same genes may pose genetic vulnerabilities toward multiple distinct psychiatric disorders.

After accounting for genetic relatedness, exposure to parental depression was associated with offspring externalising problems in several studies ${ }^{19-22,24,25,56}$, whereas parental anxiety $^{22}$ and overall internalising symptoms ${ }^{57}$ were unrelated to offspring externalising behaviours. Combined with the findings described above, this indicates that exposure to a depressed parent is a risk factor for both internalising and externalising behaviours. As with childhood internalising problems, the association between maternal depression and childhood externalising problems was often observed only in relation to concurrent depressive symptoms ${ }^{21,22,24}$, although one children-of-twins study reported an association between prenatal maternal depression and ADHD in 5-year-olds ${ }^{56}$. Current results mainly highlight that associations with prenatal depression in observational studies that do not control for genetic effects are likely partly explained by unmeasured $r G E$. One adoption study investigating $r G E$ reported evocative effects; birth parent depression predicted offspring externalising problems, which in turn predicted adoptive parent depression ${ }^{19}$. As well as demonstrating how genes and environment work in combination, the study highlights the bidirectional relationship between parent and offspring mental health phenotypes.

\section{Parenting behaviours}

Genetic associations between parenting and offspring externalising behaviours were scarcely investigated (Table 4). A children-of-twins study reported no genetic overlap between parental monitoring and offspring externalising problems $^{59}$, whereas an adoption study reported that birth mother personality characteristics were partially associated with offspring callous-unemotional behaviours ${ }^{60}$. Previous children-of-twins studies show that it is plausible that parents with a predisposition for negative parenting behaviours have offspring predisposed to psychopathology, and subsequently both phenotypes may share a common aetiology ${ }^{4}$. 


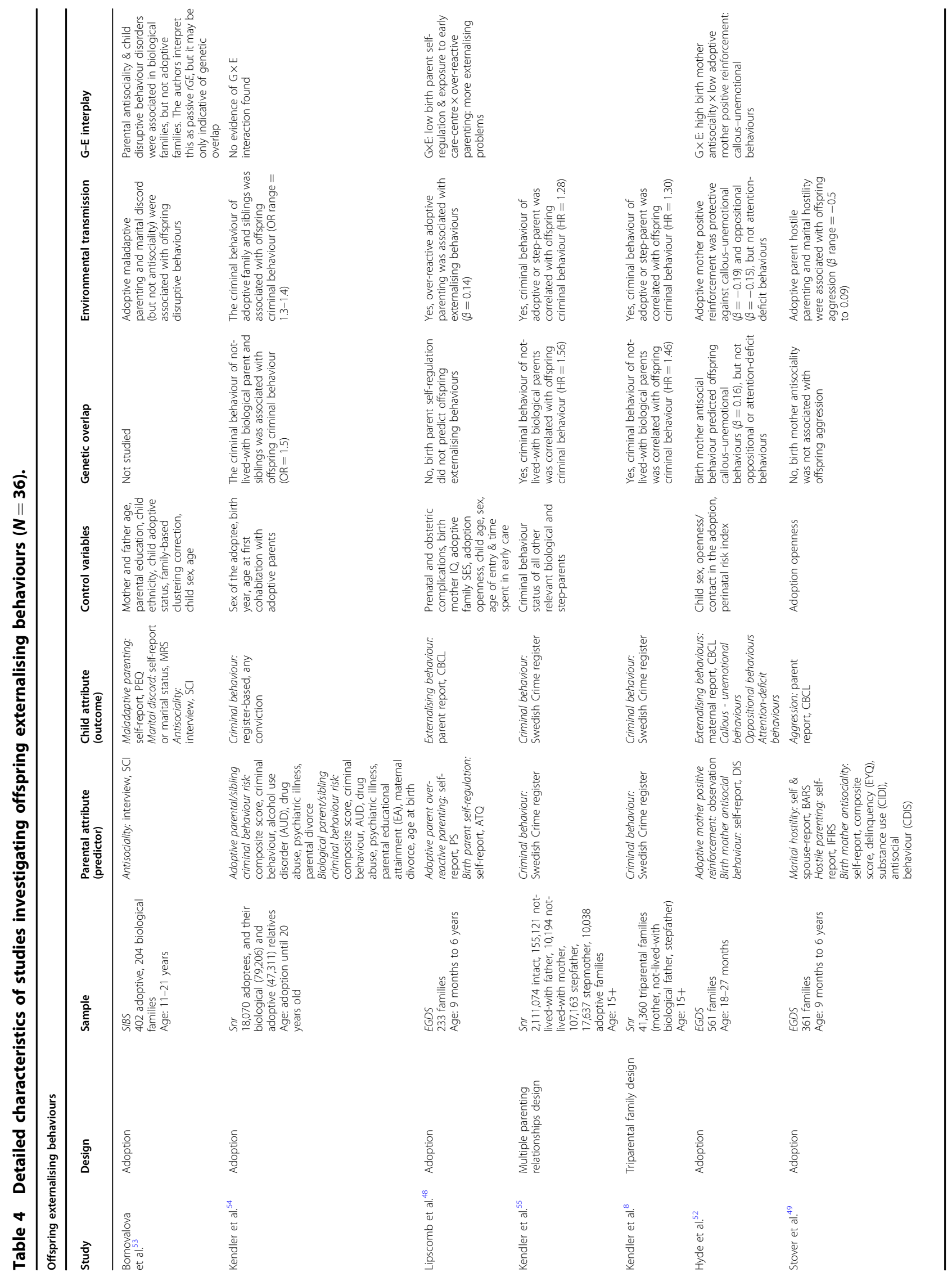




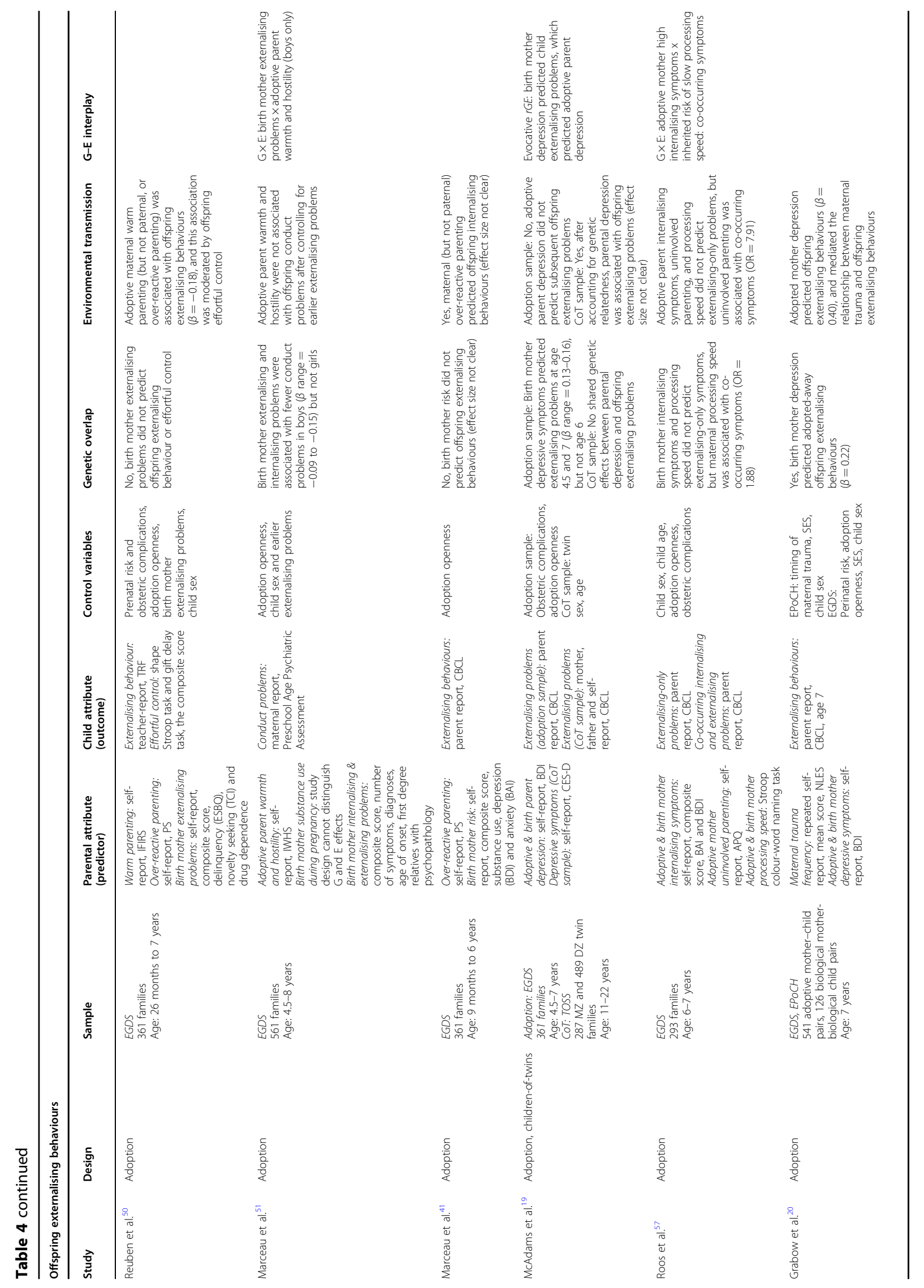




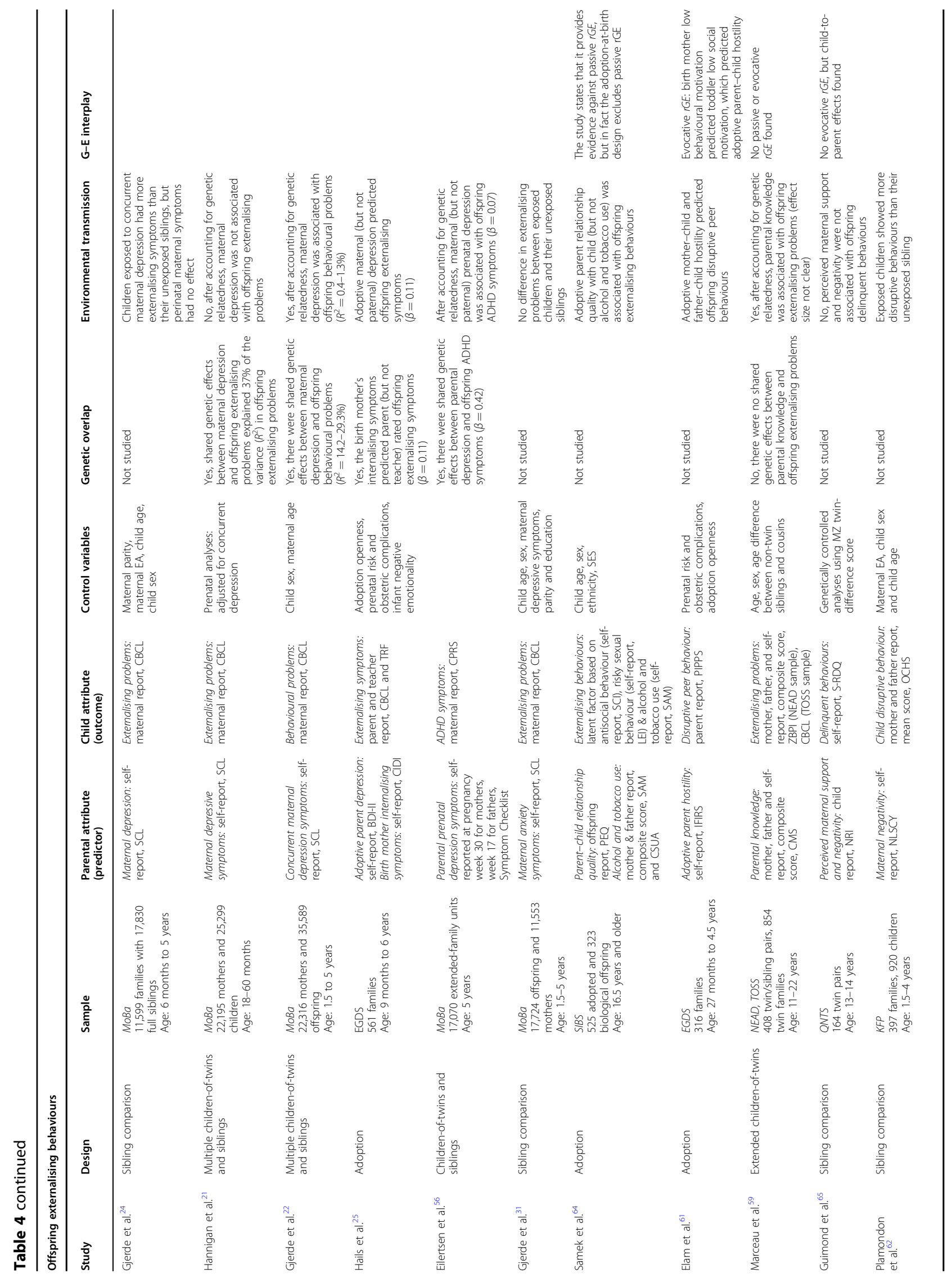




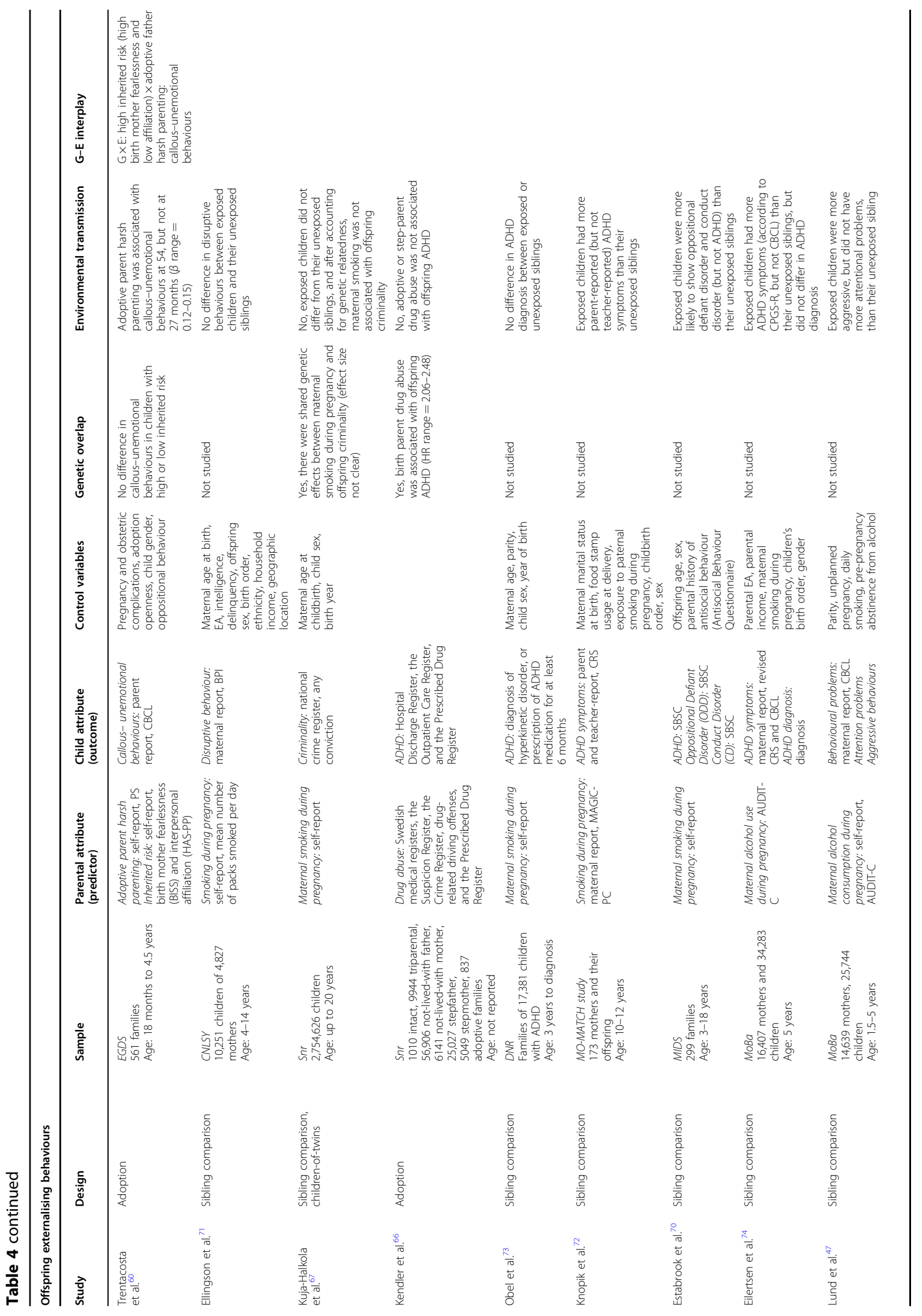




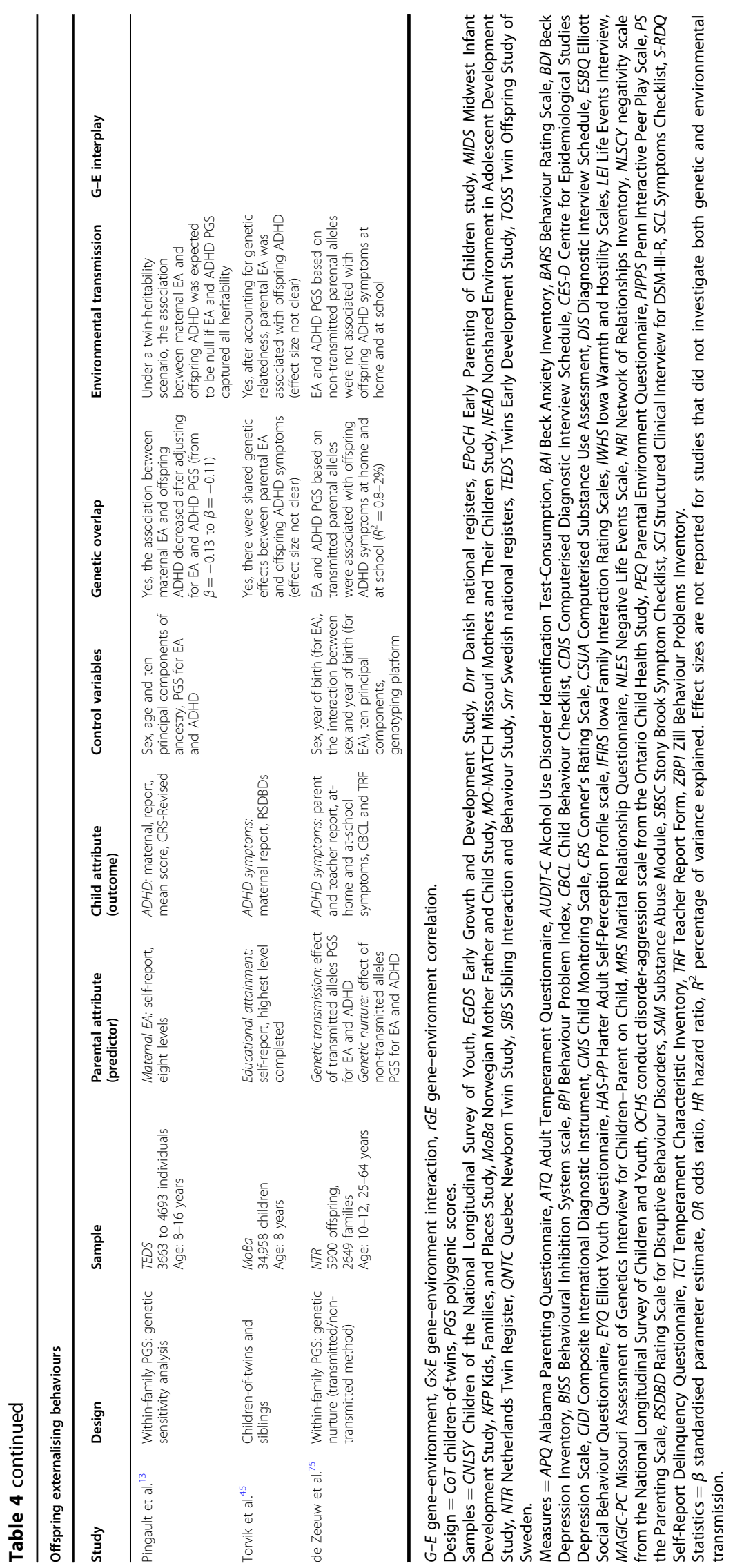


Studies of environmental transmission found associations between both positive and negative parenting and offspring externalising behaviours. Negative parenting behaviours were associated with increased offspring externalising behaviours ${ }^{49,53,60-62}$, but these effects were sometimes inconsistent across raters. For instance, overreactive parenting was associated with parent-rated ${ }^{41,48}$, but not teacher-rated ${ }^{50}$ externalising problems. This could reflect differences in the child's behaviour observed at home by the parent, or at school by the teacher. Alternatively, these differences could be indicative of rater biases resulting from differences in the interpretation of scale items, a unique perception of the children's behaviour, or the rater's own mental health ${ }^{63}$. More research is required to clarify rater-specific findings. Focusing on positive parenting, factors such as parental knowledge of offspring whereabouts, good parent-child relationship quality, positive reinforcement, and warm parenting were associated with fewer externalising problems ${ }^{50-52,59,64}$, whereas there were no associations between parental positive reinforcement and ADHD symptoms ${ }^{52}$, or maternal support and offspring delinquent behaviour ${ }^{65}$. Investigation of possible gene-environmental correlation between parenting and offspring externalising behaviours in adoption samples found no passive or evocative $r G E$ effects in the associations between parental knowledge and offspring externalising behaviours ${ }^{59}$, whereas one study reported an evocative $r G E$ showing that parental hostility was evoked by genetically influenced offspring behaviour $^{61}$, and another reported child-to-parent effects on maternal support and negativity ${ }^{65}$. As well as highlighting the bidirectionality of parent-offspring associations, these studies show that associations between parenting and offspring outcomes vary by phenotype and no single explanation fits all parenting-offspring associations.

\section{Parental substance use}

Two studies reported that parental drug abuse $e^{66}$ and smoking ${ }^{67}$ shared genetic overlap with offspring externalising behaviours (Table 4). These reports of genetic overlap are in line with classical twin studies which suggest that comorbidity between substance use and externalising behaviours is partly due to overlapping genetic factors $^{68,69}$. After accounting for genetic relatedness, mixed evidence for environmental associations between parental substance use and some offspring externalising behaviours was found. Maternal smoking during pregnancy was linked to offspring oppositional defiant disorder $^{70}$ and conduct problems ${ }^{70}$, whereas a larger study showed no association with offspring disruptive behaviours $^{71}$. Similarly, smoking during pregnancy was associated with parent-reported ADHD symptoms in one sibling comparison study ${ }^{72}$, but not another ${ }^{70}$, and was not associated with ADHD diagnosis in a large population-based sample ${ }^{73}$. Exposure to maternal alcohol use during pregnancy was linked to offspring aggression in one study ${ }^{47}$, and to offspring ADHD symptoms in another ${ }^{74}$, but the latter association was not reliably observed across measurement instruments, and moreover, maternal drinking was not associated with ADHD diagnosis $^{74}$ or attentional problems ${ }^{47}$. Studies of parental substance use during childhood found no environmental effect of parent alcohol and tobacco use ${ }^{64}$ or drug abuse ${ }^{66}$ on offspring externalising behaviours. The overall pattern of results indicates that prenatal exposure to substance use may be associated with some offspring externalising behaviours, but no firm conclusions can be drawn from current or previous work.

\section{Parental education attainment}

Three studies found evidence of genetic overlap between parental education attainment and offspring ADHD symptoms ${ }^{13,45,75}$ (Table 4). Genetic overlap between educational attainment and ADHD is previously known $^{76}$, and is hypothesised to either suggests a common neurobiological process underlying both inattention symptoms and academic achievement, or an indirect mechanism through which genetically influenced inattention impacts academic achievement ${ }^{77}$. Both of these scenarios are feasible in the context of the observed parent-offspring associations.

Findings for an environmental pathway were mixed. Although a within-family PGS study estimated that the association between maternal education and offspring ADHD would be null after adjusting for PGS that captured all heritability based on twin-based estimates ${ }^{13}$, a large children-of-twins study found that maternal education was associated with offspring ADHD symptoms even after accounting for genetic relatedness ${ }^{45}$. Parental educational attainment has been associated with specific parenting styles ${ }^{78}$, and it seems plausible that these parenting behaviours subsequently influence offspring ADHD. However, based on what we know from twin literature, where ADHD shows very high heritability, and little effects of the shared or unique environments ${ }^{3}$, the overall impact of parenting behaviours on ADHD is likely to be small.

\section{Genetic nurture}

One within-family PGS study of ADHD found no genetic nurturing effect on offspring ADHD due to ADHD or educational attainment related to parental genes $^{75}$. Although this finding requires replication, it is compatible with what we know from twin-based literature, discussed above. 


\section{Offspring educational attainment}

\section{Intergenerational transmission of educational attainment}

Studies investigating intergenerational educational attainment showed consistent evidence of genetic overlap between parent and offspring educational attainment ${ }^{13,45,79-81}$ (Table 5). Additional evidence of genetic transmission was provided by several within-family PGS studies showing that parental genetic liability for educational attainment predicted offspring educational attainment ${ }^{13,75,82-85}$. After accounting for genetic relatedness, evidence of environmental transmission of intergenerational educational attainment was observed in several studies $^{45,79-82}$. Taken together, current literature indicates that as well as passing on education-associated genes, parents may shape the rearing environment in a way that influences the offspring's subsequent educational attainment. However, these environment influences may nonetheless be partly influenced by parental genes. In line with this, a within-family PGS study provided evidence of passive $r G E$, showing that individuals with higher PGS for educational attainment tended to grow up in bettereducated households than those with lower PGS ${ }^{86}$.

\section{Genetic nurture}

Research into genetic nurture has gained traction in the last two years, starting with the publication of three landmark studies with novel designs to identify genetic nurturing effects on offspring educational attainment ${ }^{14,83,87}$ (Table 5). These studies have highlighted that parental genes can have an indirect (environmentally mediated) effect on offspring educational attainment through parental traits that are genetically influenced. The genetic nurturing effect on offspring educational attainment has been replicated in several samples ${ }^{15,75,78,84-86,88,89}$, and a few studies reported that the observed effect was partly explained by family socioeconomic status ${ }^{14,15,89}$. This finding is compatible with an adoption study which found that adoptive parents with higher income had offspring with increased educational attainment ${ }^{90}$. Other studies reported additional mediating effects of parental $\mathrm{IQ}^{88}$, maternal health during pregnancy ${ }^{89}$ and parenting behaviours ${ }^{78}$. The last study was the first to show that specific parenting behaviours are under the genetic influence of education-associated genes, and that these genetically influenced parenting behaviours are subsequently associated with offspring educational attainment. In addition, the study reported evidence of passive $r G E$, as mothers with higher PGS for education attainment provided home environments that were more conducive to higher educational attainment (greater cognitive stimulation, more warm and sensitive parenting, and less chaotic and safer, tidier homes) ${ }^{78}$. Evidence of passive $r G E$ was also found for the overall genetic nurturing effect in a within-family PGS study of adoption samples, where parental PGS of educational attainment was more strongly associated with offspring educational attainment in biological families than adoptive families ${ }^{85}$. This particular passive $r G E$ has also been reported outside of the reviewed work $^{91}$.

\section{Maternal smoking during pregnancy}

A large children-of-twins study reported genetic overlap between maternal smoking during pregnancy and offspring general cognitive ability ${ }^{67}$ (Table 5). This finding is in line with the known negative genetic correlation between smoking and educational attainment ${ }^{92}$ and highlights that in observational studies without genetically informative designs, this parent-offspring association explained by unmeasured genetic effects could lead to spurious conclusions. Investigations of environmental transmission did not reveal robust associations; maternal smoking during pregnancy was negatively associated with reading $\operatorname{cognition}^{71}$, but associations with other measures of cognitive functioning $^{71}$, general cognitive ability ${ }^{67}$, and academic achievement ${ }^{67}$ did not remain after accounting for genetic relatedness. Previous literature on genetically informative designs suggests that familial factors, including genetic effects, account for the relationship between smoking during pregnancy and offspring cognition ${ }^{93}$.

\section{Offspring substance use}

Intergenerational transmission of substance use behaviours

Studies investigating intergenerational transmission of substance use behaviours (Table 6) showed consistent evidence of genetic transmission of substance involvement $^{94}$, alcohol use ${ }^{9,95-97}$, drug abuse $\mathrm{e}^{8,9,98,99}$ and smoking initiation $^{100}$. There was also evidence of environmental transmission of many substance use behaviours, including drinking behaviour ${ }^{101}$, alcohol use disorder ${ }^{8,9,95,97}$, drug abuse $\mathrm{e}^{8,9,11,98,99,102}$, smoking behaviour ${ }^{100,103}$ and addiction-prone personalities ${ }^{104}$, whereas parental dependency on alcohol was not consistently associated with offspring alcohol involvement ${ }^{94,96}$. Two studies showed no long-term effects of maternal smoking during pregnancy on offspring substance use behaviours ${ }^{67,105}$. Although parental substance use behaviours were generally associated with an increased likelihood of substance use in offspring, an extended twin study observed negative environmental transmission of smoking behaviours, whereby parental smoking had an inhibiting effect on offspring smoking initiation ${ }^{103}$. The finding was marginally significant and requires replication. One study found evidence of passive $r G E$ underlying parent-offspring similarity in drinking behaviours, with more similarities in biological parent-child relationships than in adoptive families $^{106}$. 


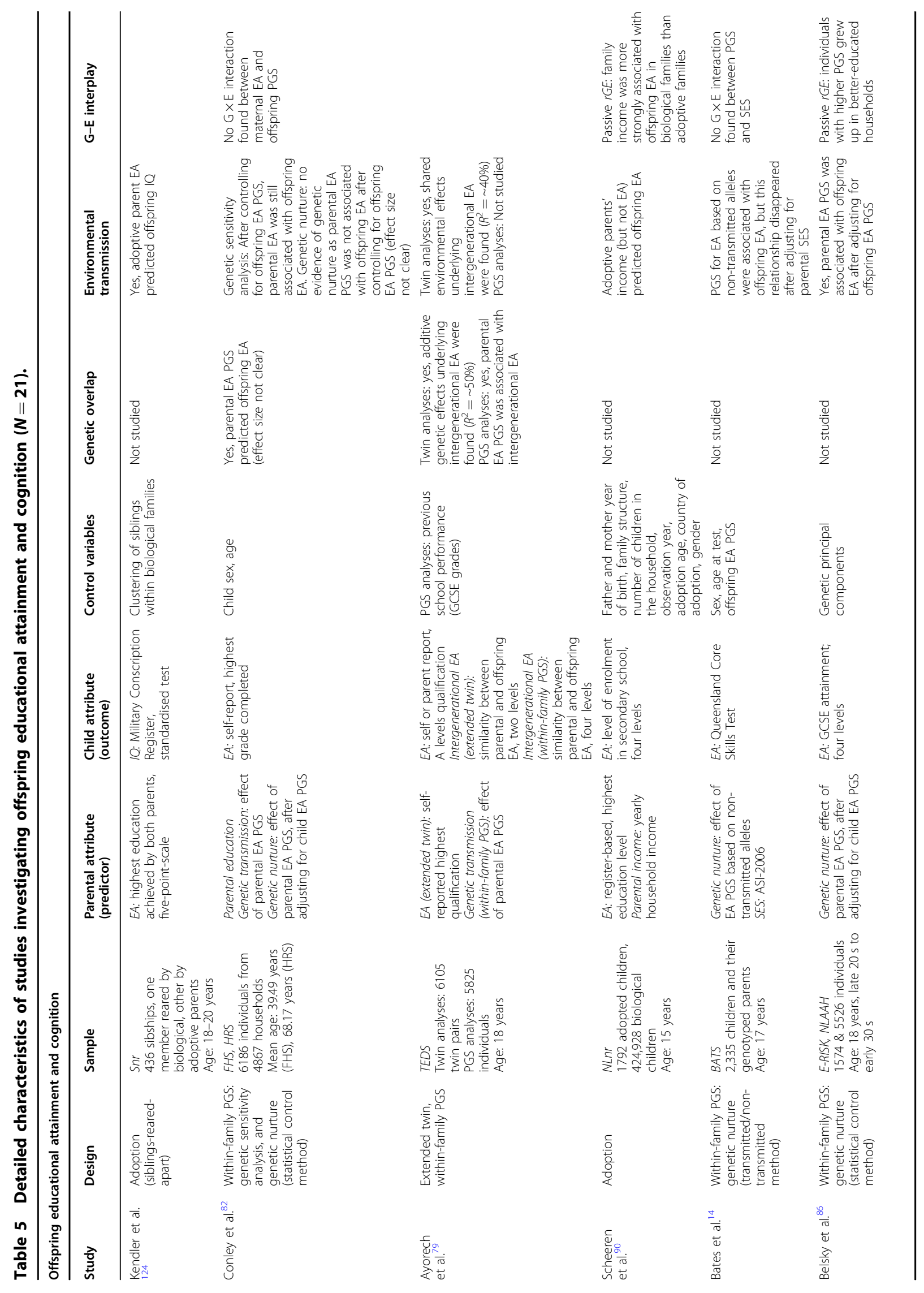




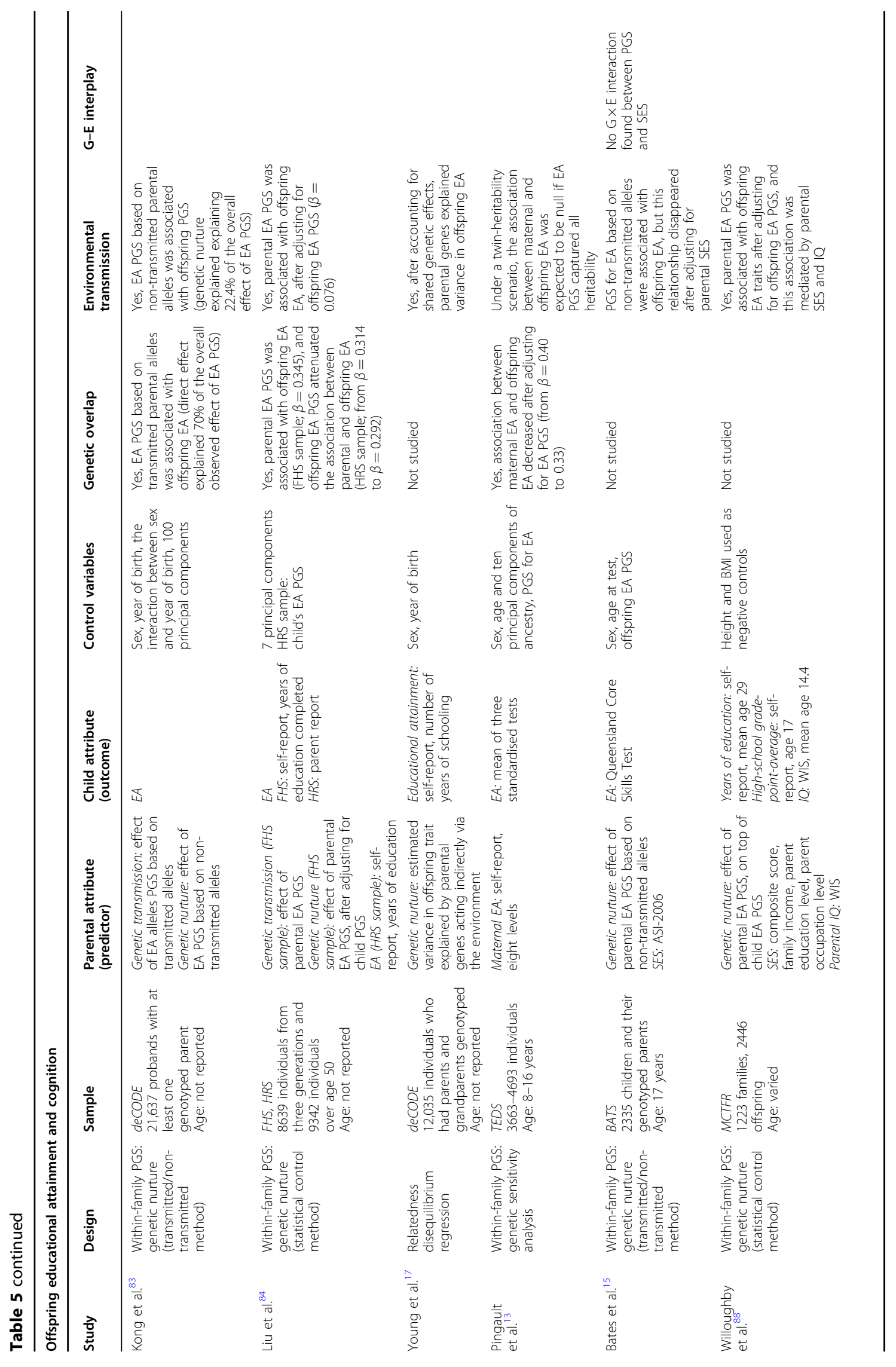




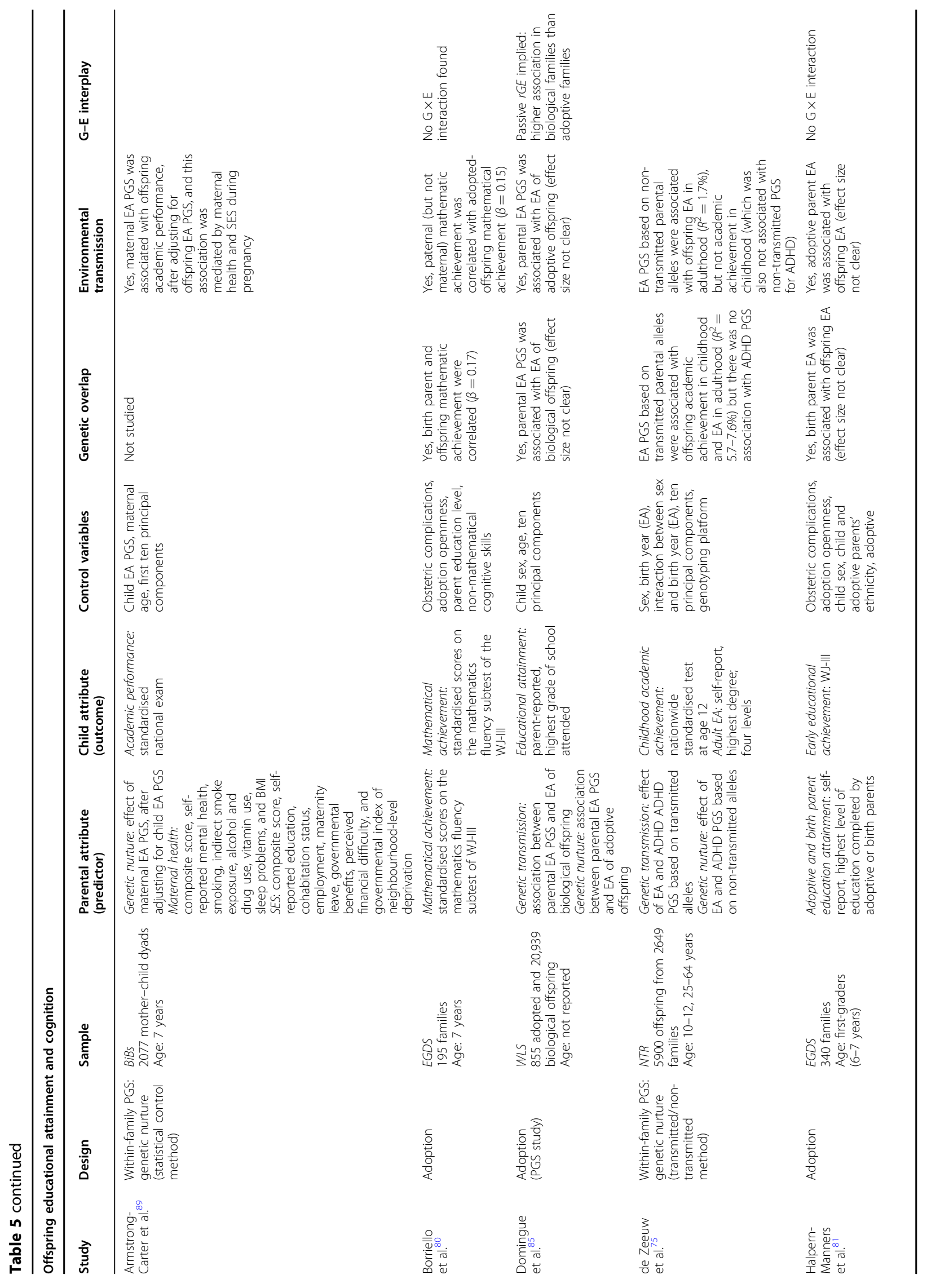




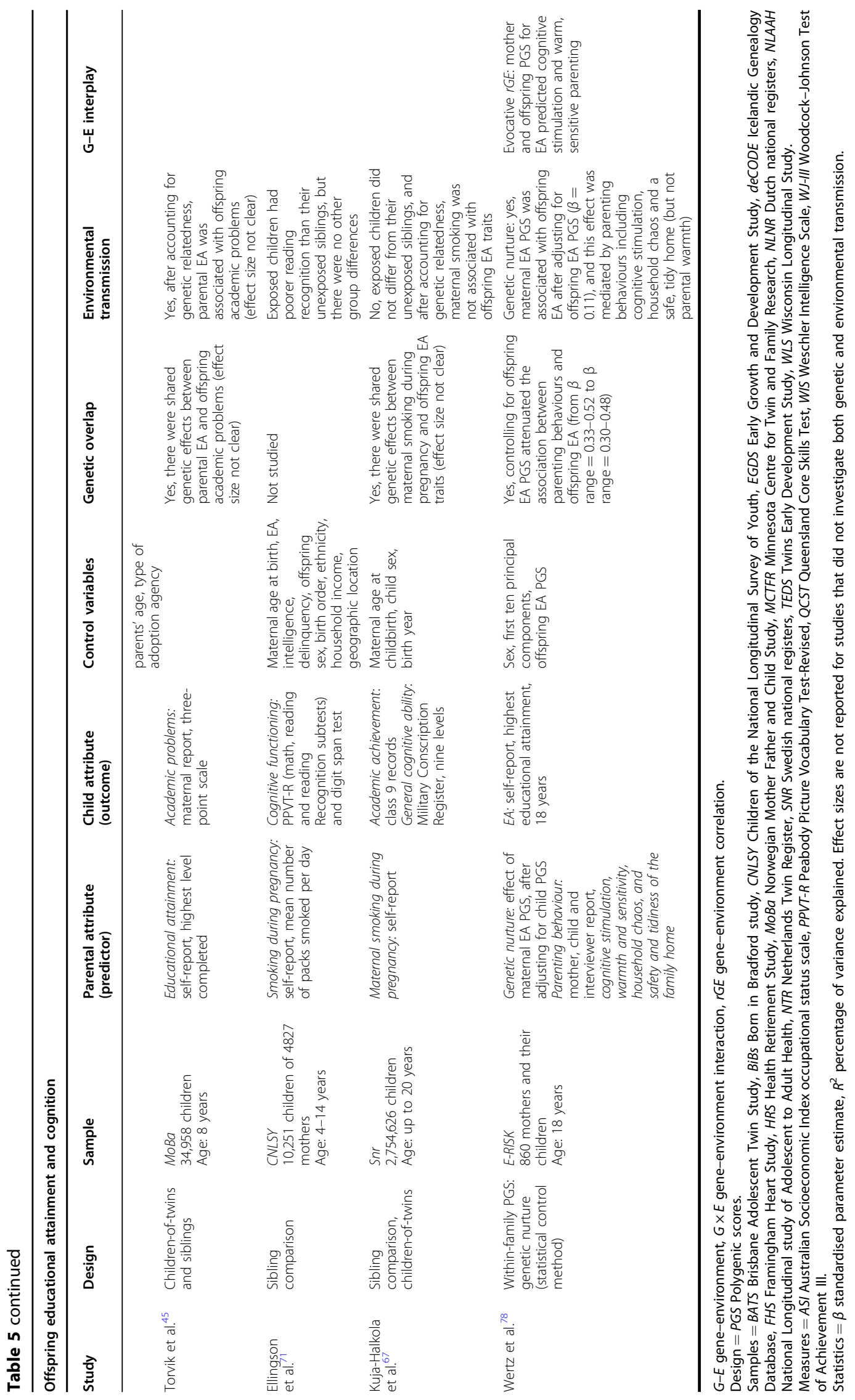




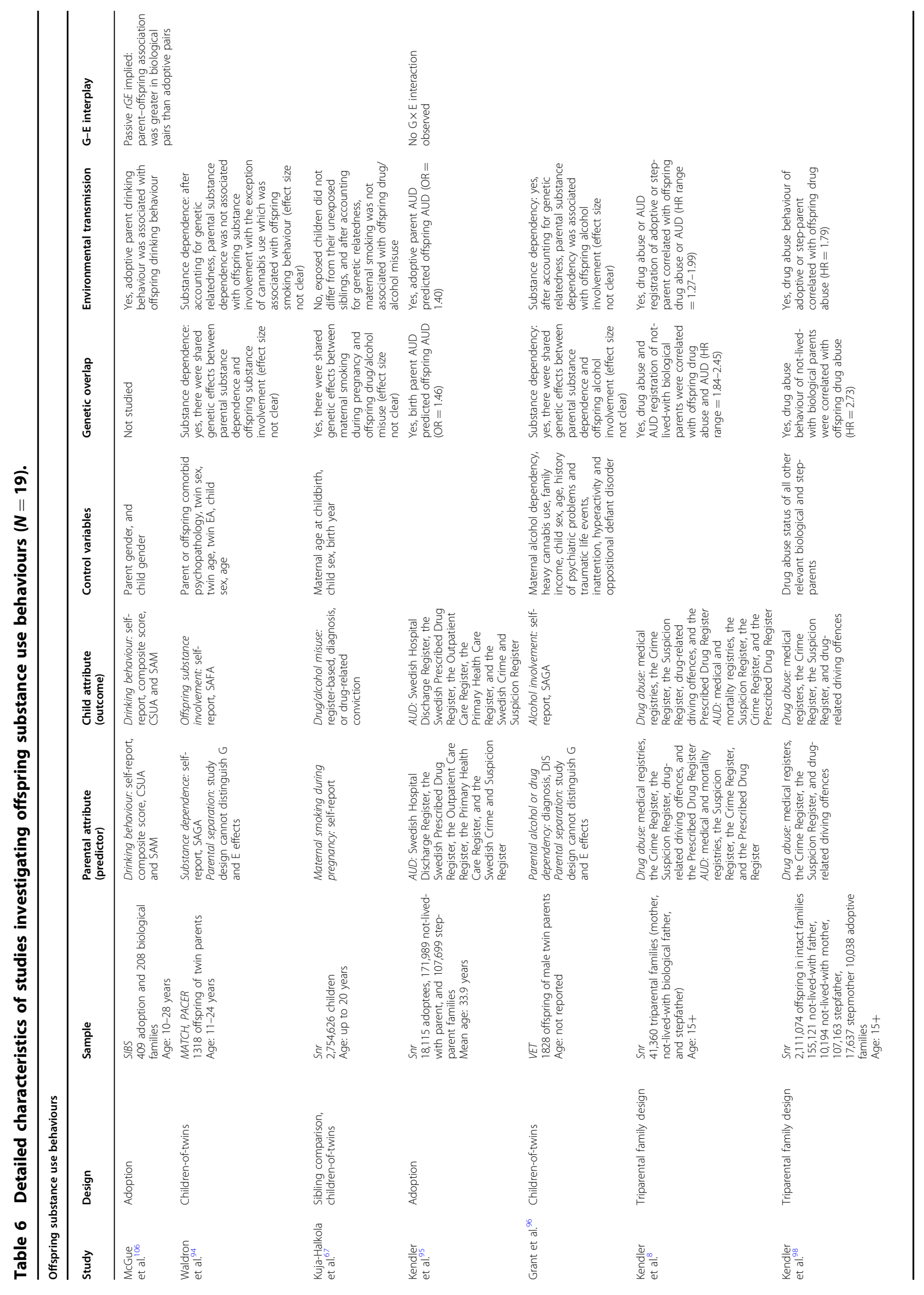




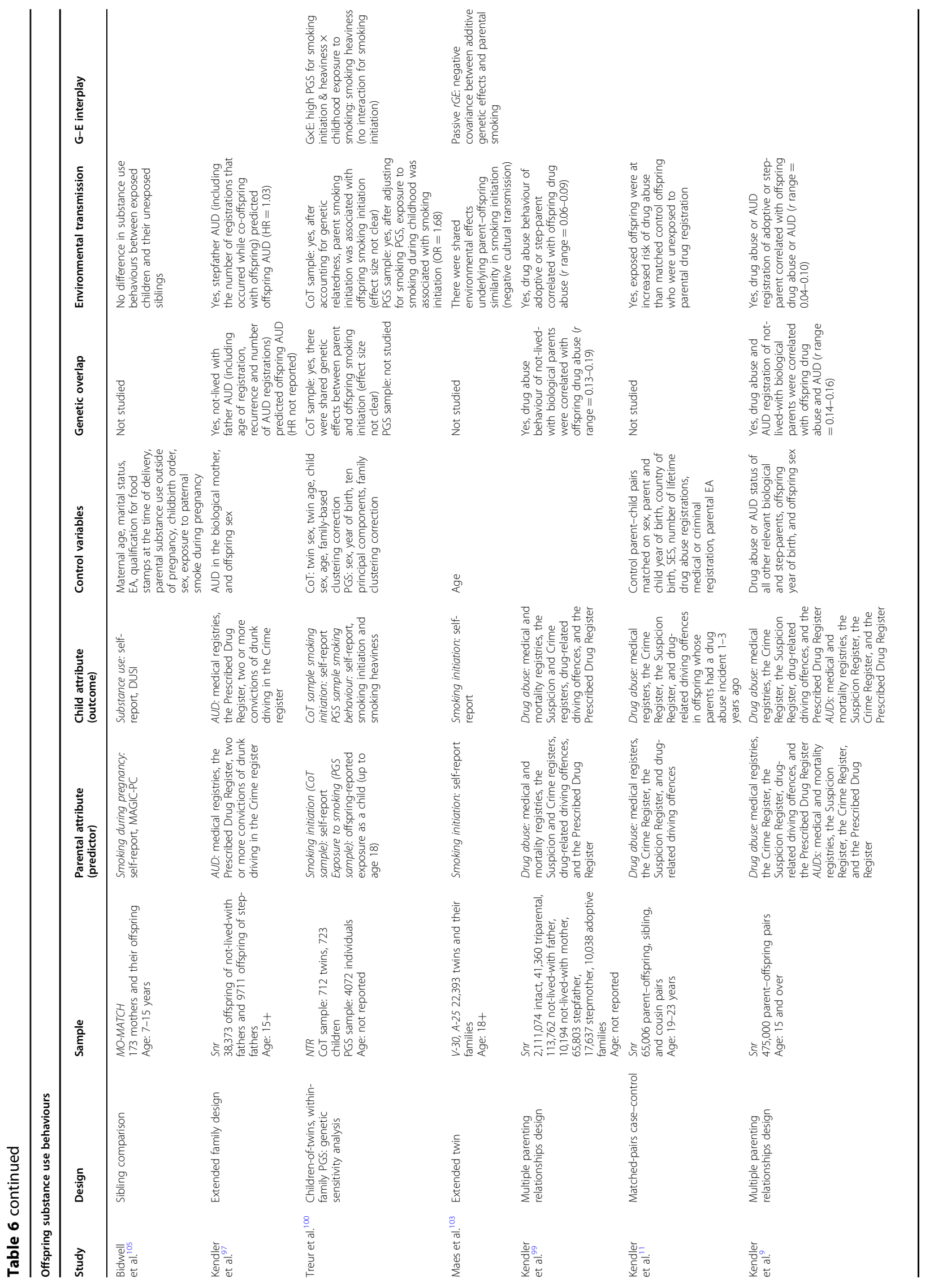




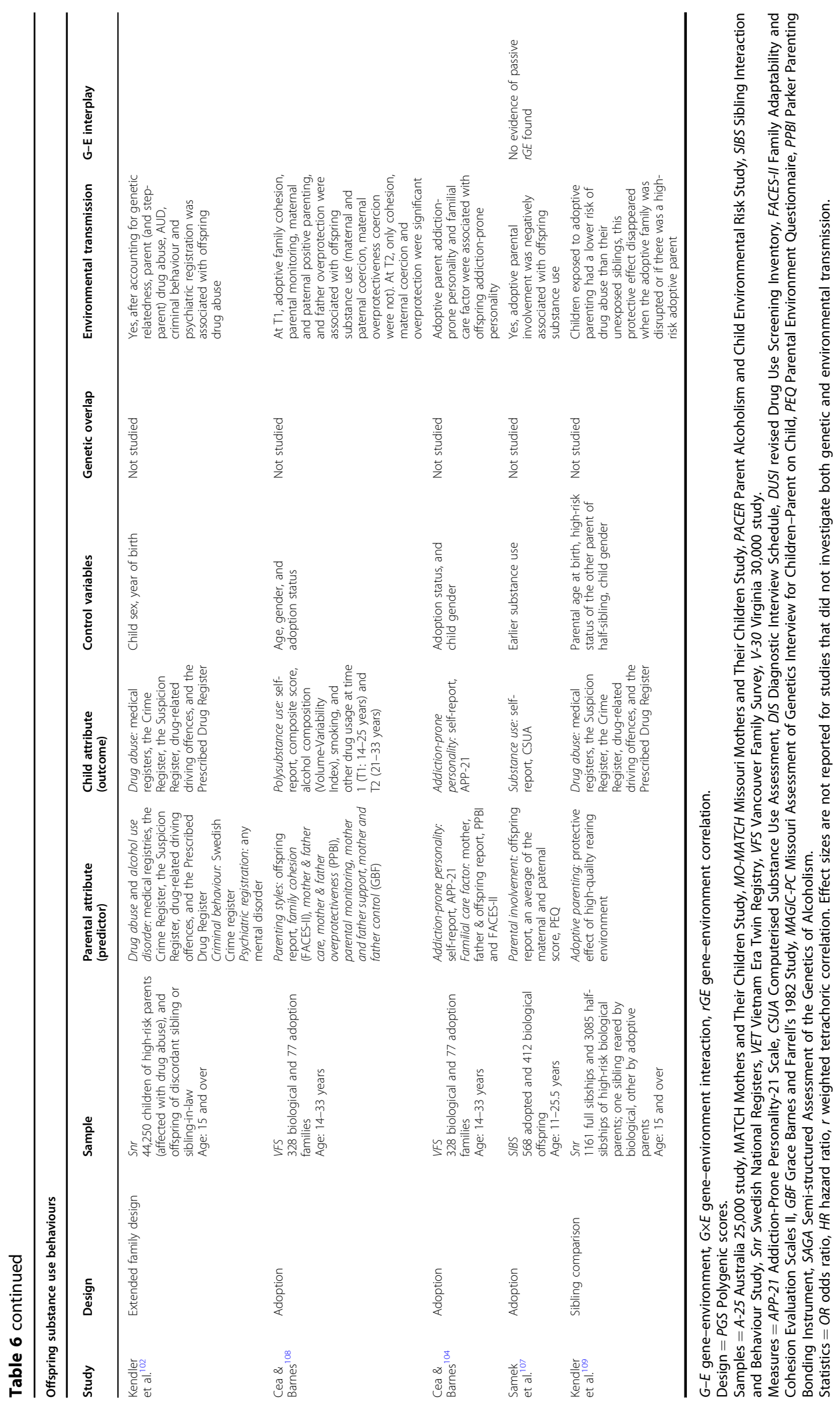




\section{Parenting behaviours}

Studies investigating the associations between parenting behaviours and offspring substance use (Table 6) showed that adoptive parenting behaviours such as parental involvement ${ }^{107}$, family care ${ }^{104}$, family cohesion, parental monitoring, parental care and parental support ${ }^{108}$ were associated with a lowered risk of offspring substance use behaviours, whereas adoptive parents' overprotectiveness or control had no effect ${ }^{108}$. In addition, children exposed to adoptive parenting had a lower risk of drug abuse than their unexposed sibling, indicating a protective effect of adoptive parenting on substance use behaviours, which was also reported for MDD above ${ }^{109}$.

\section{Offspring personality}

There was evidence of genetic and environmental influences underlying associations between parental characteristics and offspring personality (Table 7). Parent sociability and offspring positive emotionality ${ }^{110}$, and parent behavioural motivation and offspring social motivation $^{61}$ shared common genetic factors, whereas the intergenerational transmission of neuroticism seemed to be environmentally explained ${ }^{29}$. There was no evidence of an environmental association between parental traits, including anxiety ${ }^{111}$, sociability ${ }^{110}$, and smoking during pregnancy ${ }^{71}$, and offspring personality traits such as sociability and temperament. In addition, an extended twin study found no evidence of environmental transmission or $r G E$ underlying associations between parent and offspring dimensional personality traits ${ }^{112}$. However, two studies observed evocative effects of offspring social behaviours on parenting; adopted offspring's genetically influenced social behaviours predicted adoptive parent hostility ${ }^{61}$ and child-centred parenting ${ }^{111}$. Overall, current and previous literature indicates that relationships between parental factors and offspring personality vary substantially by phenotype, and can involve both genetic and environmental processes.

\section{Discussion}

This review provides a broad overview of genetically informative literature investigating associations between parental characteristics and offspring mental health and related outcomes. This is a topic of substantial interest, with 89 relevant articles published in the past 6 years. Overall, reviewed studies showed reliable evidence of genetic transmission of depression, criminal behaviour, educational attainment, and substance use behaviours from parent-tochild. Additionally, cross-phenotype genetic overlap was observed in several instances; for example, parental depression, substance use, and educational attainment were all associated with offspring externalising behaviours through genetic pathways (Table 2). After accounting for genetic transmission, parental depression or anxiety were associated with offspring internalising or externalising behaviours through environmental pathways. For maternal exposures, these associations were related to concurrent maternal symptoms, with no long-lasting effect of prenatal depression or anxiety on offspring mental health. Other environmental associations and $r G E s$ were observed for parent-offspring similarity in criminal behaviours, substance use behaviours, and educational attainment. In addition, positive and negative parenting behaviours held associations with offspring internalising behaviours, externalising behaviours, substance use behaviours, and educational attainment, with some evidence of $r G E$. Finally, cross-lagged studies showed bidirectional associations between parenting traits and offspring behaviours, where parenting predicted offspring behaviours, and offspring behaviours predicted parenting.

The reviewed literature highlights that genetically informative designs must be implemented to model or control for genetic effects in studies investigating parental influences on offspring development. There was substantial evidence of genetic overlap between parental and offspring phenotypes for both similar traits (e.g. parental depression and offspring internalising symptoms) ${ }^{19-23}$ and dissimilar traits (e.g. parental depression and offspring externalising problems) $)^{19-22,56}$. As well as indicating genetic transmission of similar traits, these findings indicate that the same genetic factors may be relevant for the development of several distinct mental health problems ${ }^{92}$, and could also partly explain the comorbidity between mental health disorders that is widely observed in literature ${ }^{113}$. Without accounting for genetic transmission within families, observational studies run a serious risk of misinterpreting these associations as causal environmental influences. For instance, it was observed that after accounting for shared genetic effects, perinatal maternal depression did not hold any long-lasting associations with offspring internalising or externalising behaviours in childhood ${ }^{21,22,24,31,34}$. This is in contrast to the substantial body of literature that interprets associations between perinatal maternal distress and offspring mental health outcomes in causal terms ${ }^{35}$. We urge future studies investigating parent-offspring associations to err on the side of caution in interpreting their results and consider evidence from multiple methodologies in forming their conclusions. Even genetically informative designs can be skewed towards non-genetic findings if there is insufficient power in the study. Triangulating evidence from multiple methodologies is required before a general conclusion can be reached on whether a given parent-offspring association is likely to be truly present, after accounting for shared genetic effects or $r G E$.

Even so, the reviewed studies indicate that both genetic and environmental factors are important in associations between parental factors and offspring mental health outcomes (Table 2). These overall findings raise two important 


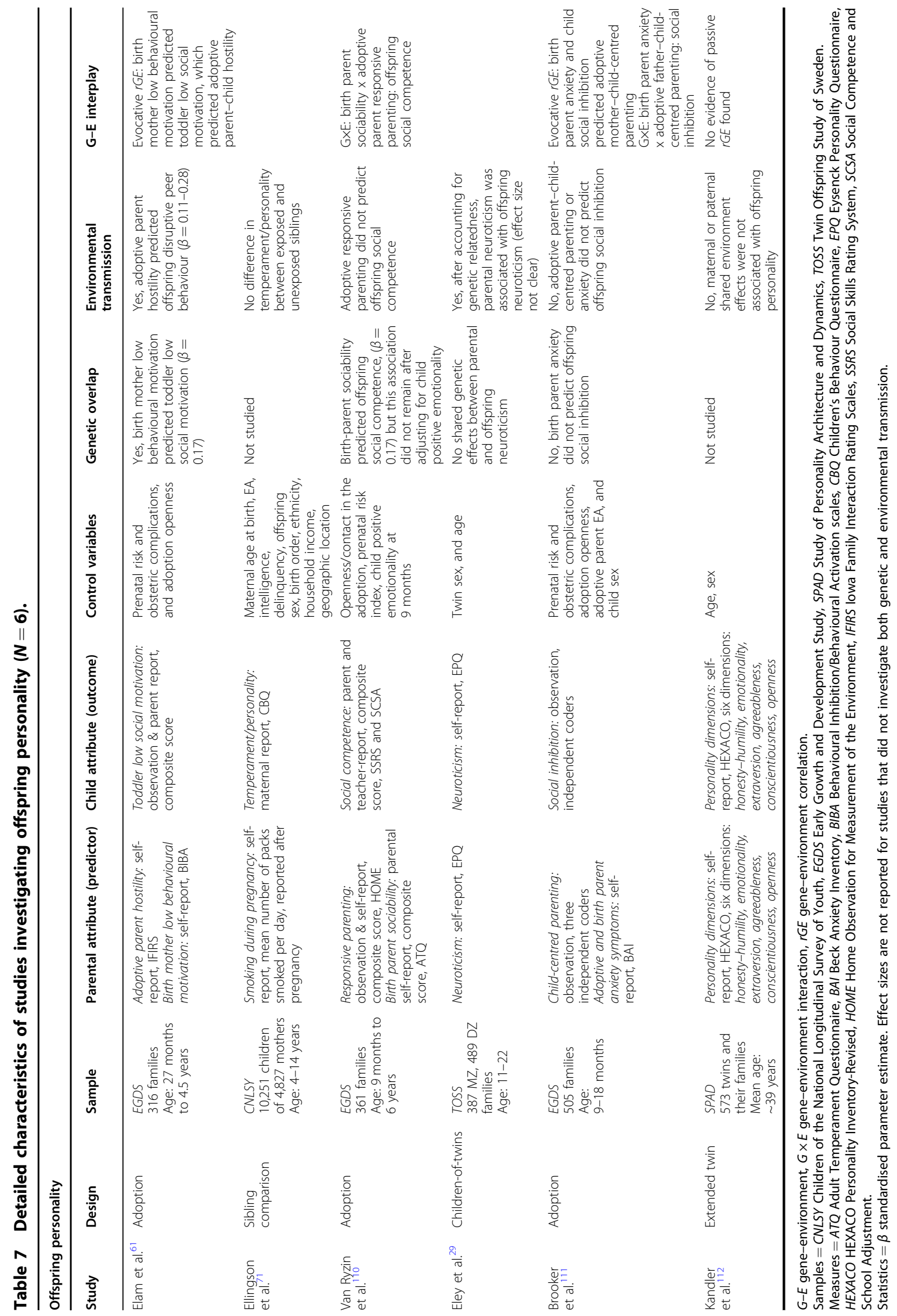


questions; to what extent are parent-offspring associations due to genetic transmission, and to what extent does parenting truly matter? Findings from classical twin literature indicate that between 40 and $80 \%$ of individual differences in mental health phenotypes such as internalising and externalising problems between people are explained by additive genetic effects ${ }^{3}$. This suggests that the largest way through which parents influence offspring mental health outcomes is through the passing on of their genes. In addition, estimates of heritability for mental health phenotypes within classical twin literature tend to increase with age, while the influence of the shared family environment decreases ${ }^{114}$. From a developmental perspective, this indicates that genetic influences on offspring mental health become increasingly important as the child gets older while the overall environmental impact of parental characteristics on offspring behaviour is likely to be small. In the current review, effect sizes showing the relative contribution of genetic and environmental factors in parent-offspring associations were not consistently reported and the available statistics are hard to compare between studies. Some studies reported higher effect sizes for genetic or environmental transmission, while others reported equal effect sizes for genetic and environmental effects in parent-offspring associations (Tables 3-7). Based on prior knowledge, the overall effect of any single parental environmental exposure is likely to be far lower than the estimated heritability of offspring mental health and related traits, as is the effect of a single genetic variant. It is also worth highlighting that environmentally mediated influences can still be under the influence of parental genes. Previous twin literature shows that parenting behaviours are under genetic influence themselves and reflect heritable individual differences ${ }^{115-117}$. Genetic nurture is a new way to index the environmentally mediated effect of parental genes on offspring behaviour. The reviewed studies provide evidence of genetic nurture effects on offspring internalising symptoms and educational attainment (Table 2). This is a promising area of research and we expect the development and application of genetic nurture designs to continue to expand in the coming years.

As well as demonstrating genetic overlap and environmental transmission within parent-offspring associations, the reviewed studies showed that confounding by passive $r G E$ is also prevalent within genetically informative designs (Table 2). If unmodelled, these unmeasured effects may inflate the estimation of both genetic and environmental factors. Additionally, evocative $r G E$ can also explain parent-offspring associations. The reviewed studies showed evidence of evocative $r G E s$ underlying associations between parental characteristics and offspring internalising symptoms, externalising symptoms and personality (Table 2). These findings are compatible with previous literature which shows a moderate impact of offspring's genetically influenced behaviours on parenting factors ${ }^{118,119}$. In instances where evocative $r G E$ effects were not observed, child-to-parent effects were sometimes still present ${ }^{19,28,30,32,36,65}$. These findings highlight the bidirectional and dynamic nature of parent-offspring associations, with child-to-parent effects, as well as parent-to-child effects, and also show the importance of cross-lagged models in modelling parent-offspring associations over time.

Reviewed findings with clinical implications are worth highlighting further. Parents with depression, anxiety, substance use problems, and externalising behaviours appeared to pass on these traits to the offspring through both genetic and environmental mechanisms. This information can be used to extend preventative and early intervention services to high-risk children of parents with internalising, externalising, or substance use disorders in healthcare settings. Family-based interventions, including cognitive, behavioural, and psychoeducational components, are already shown to be effective in children of parents with internalising and externalising disorders ${ }^{120}$. In addition, several reviewed studies showed that positive parental environments, such as parental warmth and positive reinforcement, were protective against externalising and substance use behaviours in children with high inherited risk ${ }^{51,52,109}$. Whilst preventative interventions for externalising problems already include a family component, current preventative strategies for substance use incorporate schoolbased and skills training approaches ${ }^{121}$. A family-based approach could be a valuable addition to preventative interventions of substance use behaviours in early life.

To conclude, parental factors are important predictors of offspring mental health and related outcomes. Both genetic and environmental processes are important in these associations. Further clarification of these processes requires more research. Exciting opportunities for parent-offspring research are increasingly present, with the availability of more datasets and ongoing advances in methodologies.

\section{Acknowledgements \\ This work was supported by the European Union's Horizon 2020 research and innovation programme, Marie Sklodowska Curie Actions-MSCA-ITN-2016- Innovative Training Networks (grant number 721567; CAPICE project). Eshim S. Jami was supported by an Academy Ter Meulen grant from the Royal Netherlands Academy of Arts and Sciences, and Anke R. Hammerschlag was supported by the Children's Hospital Foundation and University of Queensland strategic funding. Meike Bartels is supported by a European Research Council consolidator grant (grant number 771067 WELL-BEING). The authors thank Zainab Humayun for the illustrations used in Figs. 1 and 2.}

\section{Author details}

${ }^{1}$ Department of Biological Psychology, Vrije Universiteit Amsterdam, Amsterdam, the Netherlands. ${ }^{2}$ Department of Clinical, Educational and Health Psychology, Division of Psychology and Language Sciences, University College London, London, UK. ${ }^{3}$ Amsterdam Public Health Research Institute, Amsterdam University Medical Centres, Amsterdam, the Netherlands. ${ }^{4}$ Child Health Research Centre, University of Queensland, Brisbane, QLD, Australia. ${ }^{5}$ Child and Youth Mental Health Service, Children's Health Queensland Hospital and Health Service, Brisbane, QLD, Australia 


\section{Conflict of interest}

The authors declare no competing interests.

\section{Publisher's note}

Springer Nature remains neutral with regard to jurisdictional claims in published maps and institutional affiliations.

Received: 3 April 2020 Revised: 19 February 2021 Accepted: 3 March 2021 Published online: 01 April 2021

\section{References}

1. McLaughlin, K. A. et al. Parent psychopathology and offspring mental disorders: results from the WHO World Mental Health Surveys. Br. J. Psychiatry 200, 290-299 (2012).

2. Martin, N. G. \& Eaves, L. J. The genetical analysis of covariance structure Heredity 38, 79-95 (1977)

3. Polderman, T. J. C. et al. Meta-analysis of the heritability of human traits based on fifty years of twin studies. Nat. Genet. 47, 702 (2015).

4. McAdams, T. A. et al. Accounting for genetic and environmental confounds in associations between parent and child characteristics: a systematic review of children-of-twins studies. Psychol. Bull. 140, 1138 (2014).

5. Cadoret, R. J. Adoption studies. Alcohol Health Res. World 19, 195 (1995).

6. McAdams, T. A. et al. Revisiting the children-of-twins design: improving existing models for the exploration of intergenerational associations. Behav. Genet. 48, 397-412 (2018).

7. Thapar, A. et al. Prenatal smoking might not cause attention-deficit/hyperactivity disorder: evidence from a novel design. Biol. Psychiatry 66, 722-727 (2009).

8. Kendler, K. S., Ohlsson, H., Sundquist, J., Sundquist, K. \& Triparental Families: a new genetic-epidemiological design applied to drug abuse, alcohol use disorders, and criminal behavior in a swedish national sample. Am. J. Psychiatry 172, 553-560 (2015).

9. Kendler, K. S., Ohlsson, H., Sundquist, J. \& Sundquist, K. Parent-offspring transmission of drug abuse and alcohol use disorder: application of the multiple parenting relationships design. Am. J. Med. Genet. Part B Neuropsychiatr. Genet. 180, 249-257 (2019).

10. Lahey, B. B. \& D'Onofrio, B. M. All in the family: comparing siblings to test causal hypotheses regarding environmental influences on behavior. Curr. Directions Psychol. Sci. 19, 319-323 (2010).

11. Kendler, K. S., Ohlsson, H., Sundquist, J. \& Sundquist, K. A contagion model for within-family transmission of drug abuse. Am. J. Psychiatry 176, 239-248 (2019).

12. Wray, N. R. et al. Research review: polygenic methods and their application to psychiatric traits. J. Child Psychol. Psychiatry 55, 1068-1087 (2014).

13. Pingault, J.-B. et al. Genetic sensitivity analysis: adjusting for genetic confounding in epidemiological associations. Preprint at https:/www.bioniv. org/content/10.1101/592352v2 (2020).

14. Bates, T. C. et al. The nature of nurture: using a virtual-parent design to test parenting effects on children's educational attainment in genotyped families. Twin Res. Hum. Genet. 21, 73-83 (2018).

15. Bates, T. C. et al. Social competence in parents increases children's educational attainment: replicable genetically-mediated effects of parenting revealed by non-transmitted DNA. Twin Res. Human Genet. 22, 1-3 (2019).

16. Eaves, L. J., Pourcain, B. S., Smith, G. D., York, T. P. \& Evans, D. M. Resolving the effects of maternal and offspring genotype on dyadic outcomes in genome wide complex trait analysis ("M-GCTA"). Behav. Genet. 44, 445-455 (2014).

17. Young, A. I. et al. Relatedness disequilibrium regression estimates heritability without environmental bias. Nat. Genet. 50, 1304-1310 (2018).

18. Eilertsen, E. M. et al. Direct and indirect effects of maternal, paternal, and offspring genotypes: Trio-GCTA. Behav. Genet. 51, 154-161 (2021).

19. McAdams, T. A. et al. The relationship between parental depressive symptoms and offspring psychopathology: evidence from a children-of-twins study and an adoption study. Psychol. Med. 45, 2583-2594 (2015).

20. Grabow, A. P. et al. Using an adoption-biological family design to examine associations between maternal trauma, maternal depressive symptoms, and child internalizing and externalizing behaviors. Dev. Psychopathol. 29, 1707-1720 (2017).

21. Hannigan, L. J. et al. Maternal prenatal depressive symptoms and risk for early-life psychopathology in offspring: genetic analyses in the Norwegian Mother and Child Birth Cohort Study. Lancet Psychiatry 5, 808-815 (2018).
22. Gjerde, L. C. et al. Associations between maternal depressive symptoms and risk for offspring early-life psychopathology: the role of genetic and nongenetic mechanisms. Psychol. Med. 1-9 (2019).

23. Kendler, K. S., Ohlsson, H., Sundquist, K. \& Sundquist, J. Sources of parentoffspring resemblance for major depression in a national Swedish extended adoption study. Jama Psychiatry 75, 194-200 (2018).

24. Gjerde, L. C. et al. Maternal perinatal and concurrent depressive symptoms and child behavior problems: a sibling comparison study. J. Child Psychol. Psychiatry 58, 779-786 (2017).

25. Hails, K. A. et al. Interaction between adoptive mothers' and fathers' depressive symptoms in risk for children's emerging problem behavior. Soc. Dev. 28, 725-742 (2019).

26. Liskola, K., Raaska, H., Lapinleimu, H. \& Elovainio, M. Parental depressive symptoms as a risk factor for child depressive symptoms; testing the social mediators in internationally adopted children. Eur. Child Adolesc. Psychiatry 27, 1585-1593 (2018).

27. Brooker, R. J. et al. Birth and adoptive parent anxiety symptoms moderate the link between infant attention control and internalizing problems in toddlerhood. Dev. Psychopathol. 26, 347-359 (2014).

28. Brooker, R. J. et al. Associations between infant negative affect and parent anxiety symptoms are bidirectional: evidence from mothers and fathers. Front. Psychol. 6, https://doi.org/10.3389/fpsyg.2015.01875 (2015).

29. Eley, T. C. et al. The intergenerational transmission of anxiety: a children-oftwins study. Am. J. Psychiatry 172, 630-637 (2015).

30. Ahmadzadeh, Y. I. et al. Anxiety in the family: a genetically informed analysis of transactional associations between mother, father and child anxiety symptoms. J. Child Psychology Psychiatry Allied Disciplines. https://doi.org/ 10.1111/jcpp.13068 (2019).

31. Gjerde, L. C. et al. Maternal perinatal and concurrent anxiety and mental health problems in early childhood: a sibling-comparison study. Child Dev 91, 456-470 (2020).

32. Field, A. P. et al. Maternal and paternal influences on childhood anxiety symptoms: a genetically sensitive comparison. J. Appl. Dev. Psychol. 68, https://doi.org/10.1016/j.appdev.2020.101123 (2020).

33. Kendler, K. S., Gardner, C. O. \& Lichtenstein, P. A developmental twin study of symptoms of anxiety and depression: evidence for genetic innovation and attenuation. Psychol. Med. 38, 1567-1575 (2008).

34. Bekkhus, M. et al. Re-examining the link between prenatal maternal anxiety and child emotional difficulties, using a sibling design. Int. J. Epidemiol. 47, 156-165 (2018).

35. Glover, V. \& O'Connor, T. G. Effects of antenatal stress and anxiety: implications for development and psychiatry. Br. J. Psychiatry 180, 389-391 (2002).

36. Bridgett, D. J. et al. Contributions of mothers' and fathers' parenting to children's self-regulation: evidence from an adoption study. Dev. Sci. 21, https://doi.org/10.1111/desc.12692 (2018).

37. Horwitz, B. N. et al. Parental criticism is an environmental influence on adolescent somatic symptoms. J. Fam. Psychol. 29, 283-289 (2015).

38. McAdams, T. A. et al. Associations between the parent-child relationship and adolescent self-worth: a genetically informed study of twin parents and their adolescent children. J. Child Psychol. Psychiatry 58, 46-54 (2017).

39. Hannigan, L. J. et al. Shared genetic influences do not explain the association between parent-offspring relationship quality and offspring internalizing problems: results from a Children-of-Twins study. Psychol. Med. 48, 592-603 (2018).

40. Ahmadzadeh, Y. I. et al. Parental criticism and adolescent internalising symptoms: associations remain after accounting for shared genetic effects. Preprint at medRxiv https://doi.org/10.1101/2020.05.07.20084319 (2020).

41. Marceau, K. et al. Combined influences of genes, prenatal environment, cortisol, and parenting on the development of children's internalizing versus externalizing problems. Behav. Genet. 45, 268-282 (2015).

42. Kendler, K. S., Ohlsson, H., Sundquist, J. \& Sundquist, K. The rearing environment and risk for major depression: a Swedish national high-risk homereared and adopted-away co-sibling control study. Am. J. Psychiatry 177, 447-453 (2020).

43. Jami, E. S. et al. Maternal and paternal effects on offspring internalizing problems: results from genetic and family-based analyses. Am. J. Med. Genet. Part B Neuropsychiatr. Genet. 183, 258-267 (2020).

44. Cheesman, R. et al. How important are parents in the development of child anxiety and depression? A genomic analysis of parent-offspring trios in the Norwegian Mother Father and Child Cohort Study (MoBa). BMC Med. 18, 1-11 (2020). 
45. Torvik, F. A. et al. Mechanisms linking parental educational attainment with child ADHD, depression, and academic problems: a study of extended families in The Norwegian Mother, Father and Child Cohort Study. J. Child Psychol. Psychiatry 61, 1009-1018 (2020).

46. Jansen, P. R. et al. Polygenic scores for schizophrenia and educational attainment are associated with behavioural problems in early childhood in the general population. J. Child Psychol. Psychiatry 59, 39-47 (2018).

47. Lund, I. O. et al. Is the association between maternal alcohol consumption in pregnancy and pre-school child behavioural and emotional problems causal? Multiple approaches for controlling unmeasured confounding. Addiction 11, 1004-1014 (2019).

48. Lipscomb, S. T. et al. Genetic vulnerability interacts with parenting and early care and education to predict increasing externalizing behavior. Int. J. Behav. Dev. 38, 70-80 (2014).

49. Stover, C. S. et al. Marital hostility, hostile parenting, and child aggression: associations from toddlerhood to school age. J. Am. Acad. Child Adolesc Psychiatry 55, 235-242 (2016).

50. Reuben, J. D. et al. Warm parenting and effortful control in toddlerhood: independent and interactive predictors of school-age externalizing behavior. J. Abnorm. Child Psychol. 44, 1083-1096 (2016).

51. Marceau, K. et al. Parenting and prenatal risk as moderators of genetic influences on conduct problems during middle childhood. Dev. Psychol. $\mathbf{5 5}$ 1164-1181 (2019).

52. Hyde, L. W. et al. Heritable and nonheritable pathways to early callousunemotional behaviors. Am. J. Psychiatry 173, 903-910 (2016).

53. Bornovalova, M. A. et al. Understanding the relative contributions of direct environmental effects and passive genotype-environment correlations in the association between familial risk factors and child disruptive behavior disorders. Psychol. Med. 44, 831-844 (2014).

54. Kendler, K. S. et al. A Swedish national adoption study of criminality. Psychol. Med. 44, 1913-1925 (2014).

55. Kendler, K. S., Ohlsson, H., Morris, N. A., Sundquist, J. \& Sundquist, K. A Swedish population-based study of the mechanisms of parent-offspring transmission of criminal behavior. Psychol. Med. 45, 1093-1102 (2015).

56. Eilertsen, E. M. et al. Parental prenatal symptoms of depression and offspring symptoms of ADHD: a genetically informed intergenerational study. J. Attention Disord. https://doi.org/10.1177/1087054720914386 (2020).

57. Roos, L. E. et al. Inherited and environmental influences on a childhood cooccurring symptom phenotype: evidence from an adoption study. Dev. Psychopathol. 28, 111-125 (2016).

58. Howard, D. M. et al. Genome-wide meta-analysis of depression identifies 102 independent variants and highlights the importance of the prefrontal brain regions. Nat. Neurosci. 22, 343-352 (2019).

59. Marceau, K. et al. Parental knowledge is an environmental influence on adolescent externalizing. J. Child Psychol. Psychiatry 56, 130-137 (2015).

60. Trentacosta, C. J. et al. Callous-unemotional behaviors and harsh parenting: reciprocal associations across early childhood and moderation by inherited risk. J. Abnorm. Child Psychol. 47, 811-823 (2019).

61. Elam, K. K. et al. Adoptive parent hostility and children's peer behavior problems: examining the role of genetically informed child attributes on adoptive parent behavior. Dev. Psychol. 50, 1543-1552 (2014).

62. Plamondon, A., Browne, D. T., Madigan, S. \& Jenkins, J. M. Disentangling childspecific and family-wide processes underlying negative mother-child transactions. J. Abnorm. Child Psychol. 46, 437-447 (2018).

63. Hoyt, W. T. Rater bias in psychological research: when is it a problem and what can we do about it? Psychol. methods 5, 64 (2000).

64. Samek, D. R. et al. General and specific predictors of nicotine and alcoho dependence in early adulthood: genetic and environmental influences. J. Stud. Alcohol Drugs 75, 623-634 (2014).

65. Guimond, F. A. et al. Associations between mother-child relationship quality and adolescent adjustment: using a genetically controlled design to determine the direction and magnitude of effects. Int. J. Behav. Dev. 40, 196-204 (2016).

66. Kendler, K. S., Ohlsson, H., Sundquist, K. \& Sundquist, J. Cross-generational transmission from drug abuse in parents to attention-deficit/hyperactivity disorder in children. Psychol. Med. 46, 1301-1309 (2016).

67. Kuja-Halkola, R., D'Onofrio, B. M., Larsson, H. \& Lichtenstein, P. Materna smoking during pregnancy and adverse outcomes in offspring: genetic and environmental sources of covariance. Behav. Genet. 44, 456-467 (2014).

68. Hicks, B. M. lacono, W. G. \& McGue, M. Index of the transmissible common liability to addiction: heritability and prospective associations with substance abuse and related outcomes. Drug alcohol Depend. 123, S18-S23 (2012).
69. Kendler, K. S. \& Myers, J. The boundaries of the internalizing and externalizing genetic spectra in men and women. Psychol. Med 44, 647-655 (2014).

70. Estabrook, R. et al. Separating family-level and direct exposure effects of smoking during pregnancy on offspring externalizing symptoms: bridging the behavior genetic and behavior teratologic divide. Behav. Genet. 46, 389-402 (2016).

71. Ellingson, J. M., Goodnight, J. A., Van Hulle, C. A., Waldman, I. D. \& D'Onofrio, B. M. A sibling-comparison study of smoking during pregnancy and childhood psychological traits. Behav. Genet. 44, 25-35 (2014).

72. Knopik, V. S. et al. Smoking during pregnancy and ADHD risk: a genetically informed, multiple-rater approach. Am. J. Med. Genet. Part B Neuropsychiatr. Genet. 171, 971-981 (2016)

73. Obel, $\mathrm{C}$. et al. The risk of attention deficit hyperactivity disorder in children exposed to maternal smoking during pregnancy — a re-examination using a sibling design. J. Child Psychol. Psyciatry 57, 532-537 (2016).

74. Eilertsen, E. M. et al. Maternal alcohol use during pregnancy and offspring attention-deficit hyperactivity disorder (ADHD): a prospective sibling control study. Int. J. Epidemiol. 46, 1633-1640 (2017).

75. de Zeeuw, E. L. et al. Intergenerational transmission of education and ADHD: effects of parental genotypes. Behav. Genet. 50, 221-232 (2020).

76. Demontis, D. et al. Discovery of the first genome-wide significant risk loci for attention deficit/hyperactivity disorder. Nat. Genet. 51, 63-75 (2019).

77. Liu, C.-Y., Li, Y., Viding, E., Asherson, P. \& Pingault, J.-B. The developmental course of inattention symptoms predicts academic achievement due to shared genetic aetiology: a longitudinal twin study. Eur. Child Adolesc. Psychiatry 28, 367-375 (2019).

78. Wertz, J. et al. Using DNA from mothers and children to study parental investment in children's educational attainment. Child Dev. https://doi.org/ 10.1111/cdev.13329 (2019).

79. Ayorech, Z., Krapohl, E., Plomin, R. \& von Stumm, S. Genetic influence on intergenerational educational attainment. Psychol. Sci. 28, 1302-1310 (2017).

80. Borriello, G. A. et al. The intergenerational transmission of mathematics achievement in middle childhood: a prospective adoption design. Dev. Sci. https://doi.org/10.1111/desc.12974 (2020).

81. Halpern-Manners, A. et al. The intergenerational transmission of early educational advantages: new results based on an adoption design. Res. Soc. Stratif. Mobil. 67, https://doi.org/10.1016/j.rssm.2020.100486 (2020).

82. Conley, D. et al. Is the effect of parental education on offspring biased or moderated by genotype? J. Soc. Sci. 2, 82 (2015).

83. Kong, A. et al. The nature of nurture: effects of parental genotypes. Science 359, 424-428 (2018).

84. Liu, H. X. Social and genetic pathways in multigenerational transmission of educational attainment. Am. Soc. Rev. 83, 278-304 (2018).

85. Domingue, B. W. \& Fletcher, J. Separating measured genetic and environmental effects: evidence linking parental genotype and adopted child outcomes. Behav. Genet. https://doi.org/10.1007/s10519-020-10000-4 (2020).

86. Belsky, D. W. et al. Genetic analysis of social-class mobility in five longitudinal studies. Proc. Natl Acad. Sci. USA 115, E7275-E7284 (2018)

87. Young, A. I., Benonisdottir, S., Przeworski, M. \& Kong, A. Deconstructing the sources of genotype-phenotype associations in humans. Science 365 1396-1400 (2019)

88. Willoughby, E. A., McGue, M., lacono, W. G., Rustichini, A. \& Lee, J. J. The role of parental genotype in predicting offspring years of education: evidence for genetic nurture. Mol. Psychiatry 1-9 (2019).

89. Armstrong-Carter, E et al. The earliest origins of genetic nurture: the prenatal environment mediates the association between maternal genetics and child development. Psychol. Sci. https://doi.org/10.1177/0956797620917209 (2020).

90. Scheeren, L., Das, M. \& Liefbroer, A. C. Intergenerational transmission of educational attainment in adoptive families in the Netherlands. Res. Soc Stratif. Mobil. 48, 10-19 (2017).

91. Cheesman, R. et al. Comparison of adopted and nonadopted individuals reveals gene-environment interplay for education in the UK Biobank. Psychol. Sci. 31, 582-591 (2020)

92. Bulik-Sullivan, B. et al. An atlas of genetic correlations across human diseases and traits. Nat. Genet. 47, 1236-1241 (2015).

93. D'Onofrio, B. M. Lahey, B. B., Turkheimer, E. \& Lichtenstein, P. Critical need for family-based, quasi-experimental designs in integrating genetic and social science research. Am. J. Public Health 103, S46-S55 (2013).

94. Waldron, M. et al. Parental separation and early substance involvement: results from children of alcoholic and cannabis dependent twins. Drug Alcohol Depend. 134, 78-84 (2014). 
95. Kendler, K. S. et al. An extended Swedish National Adoption Study Of Alcohol Use Disorder. JAMA Psychiatry 72, 211-218 (2015).

96. Grant, J. D. et al. Parental separation and offspring alcohol involvement: findings from offspring of alcoholic and drug dependent twin fathers. Alcohol Clin. Exp. Res. 39, 1166-1173 (2015).

97. Kendler, K. S., Ohlsson, H., Edwards, A., Sundquist, J. \& Sundquist, K. The clinical features of alcohol use disorders in biological and step-fathers that predict risk for alcohol use disorders in offspring. Am. J. Med. Genet. Part B Neuropsychiatr. Genet. 174, 779-785 (2017).

98. Kendler, K. S., Ohlsson, H., Sundquist, K. \& Sundquist, J. The causes of parentoffspring transmission of drug abuse: a Swedish population-based study. Psychol. Med. 45, 87-95 (2015).

99. Kendler, K. S., Ohlsson, H., Sundquist, K. \& Sundquist, J. Sources of parent-child transmission of drug abuse: path analyses of not-lived-with parental, stepparental, triparental, and adoptive families. J. Nerv. Ment. Dis. 206, 239-244 (2018).

100. Treur, J. L. et al. Testing familial transmission of smoking with two different research designs. Nicotine Tob. Res. 20, 836-842 (2018).

101. McGue, M. et al. The environments of adopted and non-adopted youth evidence on range restriction from the Sibling Interaction and Behavior Study (SIBS). Behav. Genet. 37, 449-462 (2007).

102. Kendler, K. S., Ohlsson, H., Sundquist, J. \& Sundquist, K. Facilitating versus inhibiting the transmission of drug abuse from high-risk parents to their children: a Swedish National Study. Twin Res. Hum. Genet. 23, 1-7 (2020).

103. Maes, H. H. et al. Cross-cultural comparison of genetic and cultural transmission of smoking initiation using an extended twin kinship model. Twin Res. Hum. Genet. 21, 179-190 (2018).

104. Cea, N. F. \& Barnes, G. E. The development of addiction-prone personality traits in biological and adoptive families. Personal. Individ. Differences $\mathbf{8 2}$, 107-113 (2015)

105. Bidwell, L. C. et al. Prenatal exposure effects on early adolescent substance use: preliminary evidence from a genetically informed ayesian approach. J. Stud. Alcohol Drugs 78, 789-794 (2017).

106. McGue, M., Malone, S., Keyes, M. \& lacono, W. G. Parent-offspring similarity for drinking: a longitudinal adoption study. Behav. Genet. 44, 620-628 (2014).

107. Samek, D. R., Rueter, M. A., Keyes, M. A., McGue, M. \& lacono, W. G. Parent involvement, sibling companionship, and adolescent substance use: a longitudinal, genetically informed design. J. Fam. Psychol. 29, 614-623 (2015).

108. Cea, N. F. \& Barnes, G. E. Parenting styles and offspring's polysubstance use in biological and adoptive families. Int. J. Child Youth Fam. Stud. 5, 466-492 (2014).

109. Kendler, K. S., Ohlsson, H., Sundquist, K. \& Sundquist, J. The rearing environment and risk for drug abuse: a Swedish national high-risk adopted and not adopted co-sibling control study. Psychol. Med. 46, 1359-1366 (2016).
110. Van Ryzin, M. J. et al. Genetic influences can protect against unresponsive parenting in the prediction of child social competence. Child Dev. 86, 667-680 (2015).

111. Brooker, R. J. et al. Early inherited risk for anxiety moderates the association between fathers' child-centered parenting and early social inhibition. J. Dev. Orig. Health Dis. 7, 602-615 (2016).

112. Kandler, C., Richter, J. \& Zapko-Willmes, A. The nature and nurture of HEXACO personality trait differences an extended twin family study. Z. Fur Psychologie J. Psychol. 227, 195-206 (2019).

113. Lai, H. M. X., Cleary, M., Sitharthan, T. \& Hunt, G. E. Prevalence of comorbid substance use, anxiety and mood disorders in epidemiological surveys, 1990-2014: a systematic review and meta-analysis. Drug Alcohol Depend. 154, 1-13 (2015).

114. Bergen, S. E., Gardner, C. O. \& Kendler, K. S. Age-related changes in heritability of behavioral phenotypes over adolescence and young adulthood: a metaanalysis. Twin Res. Hum. Genet. 10, 423-433 (2007).

115. Plomin, R., Reiss, D., Hetherington, E. M. \& Howe, G. W. Nature and nurture: genetic contributions to measures of the family environment. Dev. Psychol. 30, 32 (1994).

116. Klahr, A. M. \& Burt, S. A. Elucidating the etiology of individual differences in parenting: a meta-analysis of behavioral genetic research. Psychol. Bull. 140, 544 (2014).

117. Vinkhuyzen, A. A. E., Van Der Sluis, S., De Geus, E. J. C., Boomsma, D. I. \& Posthuma, D. Genetic influences on 'environmental' factors. Genes, Brain Behav. 9, 276-287 (2010).

118. Avinun, R. \& Knafo, A. Parenting as a reaction evoked by children's genotype: a meta-analysis of children-as-twins studies. Personal. Soc. Psychol. Rev. 18, 87-102 (2013).

119. Kendler, K. S. \& Baker, J. H. Genetic influences on measures of the environment: a systematic review. Psychol. Med. 37, 615-626 (2007)

120. Siegenthaler, E., Munder, T. \& Egger, M. Effect of preventive interventions in mentally ill parents on the mental health of the offspring: systematic review and meta-analysis. J. Am. Acad. Child Adolesc. Psychiatry 51, 8-17 (2012).

121. Stockings, E. et al. Prevention, early intervention, harm reduction, and treatment of substance use in young people. Lancet Psychiatry 3, 280-296 (2016).

122. Keller, M. C. et al. Modeling extended twin family data I: description of the Cascade model. Twin Res Hum. Genet. 12, 8-18 (2009).

123. O'Reilly, L. M. et al. The intergenerational transmission of suicidal behavior: an offspring of siblings study. Transl. Psychiatry 10, 173 (2020).

124. Kendler, K. S., Turkheimer, E., Ohlsson, H., Sundquist, J. \& Sundquist, K. Family environment and the malleability of cognitive ability: a Swedish national home-reared and adopted-away cosibling control study. Proc. Natl Acad. Sci. USA 112, 4612-4617 (2015). 